\title{
The tiered Aubry set for autonomous Lagrangian functions
}

\author{
M.-C. ARNAUD *
}

November 22, 2018

\begin{abstract}
Let $L: T M \rightarrow \mathbb{R}$ be a Tonelli Lagrangian function (with $M$ compact and connected and $\operatorname{dim} M \geq 2)$. The tiered Aubry set (resp. Mañé set) $\mathcal{A}^{T}(L)\left(\right.$ resp. $\left.\mathcal{N}^{T}(L)\right)$ is the union of the Aubry sets (resp. Mañé sets) $\mathcal{A}(L+\lambda)$ (resp. $\mathcal{N}(L+\lambda)$ ) for $\lambda$ closed 1-form. Then :

1. the set $\mathcal{N}^{T}(L)$ is closed, connected and if $\operatorname{dim} H^{1}(M) \geq 2$, its intersection with any energy level is connected and chain transitive;

2. for $L$ generic in the Mañé sense, the sets $\overline{\mathcal{A}^{T}(L)}$ and $\overline{\mathcal{N}^{T}(L)}$ have no interior;

3. if the interior of $\overline{\mathcal{A}^{T}(L)}$ is non empty, it contains a dense subset of periodic points.

Then, we give an example of an explicit Tonelli Lagrangian function satisfying 2 and an example proving that when $M=\mathbb{T}^{2}$, the closure of the tiered Aubry set and the closure of the union of the K.A.M. tori may be different.
\end{abstract}

\section{Résumé}

Soit $L: T M \rightarrow \mathbb{R}$ un lagrangien de Tonelli (avec $M$ compacte et connexe et $\operatorname{dim} M \geq$ 2). L'ensemble d'Aubry (resp. de Mañé) étagé $\mathcal{A}^{T}(L)$ (resp. $\mathcal{N}^{T}(L)$ ) est la réunion des ensembles d'Aubry (resp. de Mañé) $\mathcal{A}(L+\lambda)$ (resp. $\mathcal{N}(L+\lambda))$ pour $\lambda 1$-forme fermée. On montre :

1. $\mathcal{N}^{T}(L)$ est fermé, connexe et si $\operatorname{dim} H^{1}(M) \geq 2$, sa trace avec chaque niveau d'énergie est connexe et transitive par chaîne;

\footnotetext{
*Université d'Avignon et des Pays de Vaucluse, Laboratoire d'Analyse non linéaire et Géométrie (EA 2151), F-84 018Avignon, France. e-mail: Marie-Claude.Arnaud@univ-avignon.fr
} 
2. si $L$ est générique au sens de Mañé, les ensembles $\overline{\mathcal{A}^{T}(L)}$ et $\overline{\mathcal{N}^{T}(L)}$ sont d'intérieur vide;

3. si l'intérieur de $\overline{\mathcal{A}^{T}(L)}$ est non vide, il contient une partie dense de points périodiques.

On donne ensuite un exemple explicite satisfaisant 2 et un exemple montrant que si $M=\mathbb{T}^{2}, \overline{\mathcal{A}^{T}(L)}$ peut être différent de l'adhérence de la réunion des tores K.A.M.

\section{Contents}

1 Introduction 3

2 Peierls barrier, Mañé potential, Aubry and Mañé sets, proof of proposition 1

3 Radially transformed set and Aubry set, proof of proposition 4 14

4 Green bundles, conjugate points and proofs of of theorem 2 and corol-

lary 3

5 Proof of proposition 6 


\section{Introduction}

Let $M$ be a compact and connected manifold endowed with a Riemannian metric; we assume that $\operatorname{dim} M \geq 2$. We will denote by $(x, v)$ a point of the tangent bundle $T M$ with $x \in M$ and $v$ a vector tangent at $x$. The projection $\pi: T M \rightarrow M$ is then $(x, v) \rightarrow x$. The notation $(x, p)$ will designate a point of the cotangent bundle $T^{*} M$ with $p \in T_{x}^{*} M$. and $\pi^{*}: T^{*} M \rightarrow M$ will be the canonical projection $(x, p) \rightarrow x$.

We consider a Lagrangian function $L: T M \rightarrow \mathbb{R}$ which is $C^{\infty}$ and:

- uniformly superlinear: uniformly on $x \in M$, we have: $\lim _{\|v\| \rightarrow+\infty} \frac{L(x, v)}{\|v\|}=+\infty$;

- strictly convex: for all $(x, v) \in T M, \frac{\partial^{2} L}{\partial v^{2}}(x, v)$ is positive definite.

Such a Lagrangian function will be called a "Tonelli Lagrangian function".

We can associate to such a Lagrangian function the Legendre map $\mathcal{L}=\mathcal{L}_{L}: T M \rightarrow$ $T^{*} M$ defined by: $\mathcal{L}(x, v)=\frac{\partial L}{\partial v}(x, v)$ which is a fibered $C^{\infty}$ diffeomorphism and the $C^{\infty}$ Hamiltonian function $H: T^{*} M \rightarrow \mathbb{R}$ defined by: $H(x, p)=p\left(\mathcal{L}^{-1}(x, p)\right)-L\left(\mathcal{L}^{-1}(x, p)\right)$ (such a Hamiltonian function will be called a "Tonelli Hamiltonian function"). The Hamiltonian function $H$ is then superlinear, strictly convex in the fiber and $C^{\infty}$. We denote by $\left(f_{t}^{L}\right)$ the Euler-Lagrange flow associated to $L$ and $\left(\Phi_{t}^{H}\right)$ the Hamiltonian flow associated to $H$; then we have : $\Phi_{t}^{H}=\mathcal{L} \circ f_{t}^{L} \circ \mathcal{L}^{-1}$.

If $\lambda$ is a $\left(C^{\infty}\right)$ closed 1 -form of $M$, then the map $T_{\lambda}: T^{*} M \rightarrow T^{*} M$ defined by : $T_{\lambda}(x, p)=(x, p+\lambda(x))$ is a symplectic $\left(C^{\infty}\right)$ diffeomorphism; therefore, we have : $\left(\Phi_{t}^{H \circ T_{\lambda}}\right)=\left(T_{\lambda}^{-1} \circ \Phi_{t} \circ T_{\lambda}\right)$, i.e. the Hamiltonian flow of $H$ and $H \circ T_{\lambda}$ are conjugated. Moreover, the Hamiltonian function $H \circ T_{\lambda}$ is associated to the Tonelli Lagrangian function $L-\lambda$, and it is well-known that : $\left(f_{t}^{L}\right)=\left(f_{t}^{L-\lambda}\right)$ : the two Euler-Lagrange flows are equal. Let us emphasize that these flows are equal, but the Lagrangian functions, and then the Lagrangian actions differ and so the minimizing "objects" may be different.

The reader will find the whole necessary mathematical background concerning Mather set, Aubry set and Mañé set in the section 3 ,

For a Tonelli Lagrangian function $(L$ or $L-\lambda)$, J. Mather introduced in [17] (see [15] too) a particular subset $\mathcal{A}(L-\lambda)$ of $T M$ which he called the "static set" and which is now usually called the "Aubry set" (this name is due to A. Fathi) 1 . There exist different but equivalent definitions of this set (see [8], [10, [15] and section 30) and it is known that two closed 1-forms which are in the same cohomological class define

\footnotetext{
${ }^{1}$ These sets extend the notion of "Aubry-Mather" sets for the twist maps.
} 
the same Aubry set :

$$
\left[\lambda_{1}\right]=\left[\lambda_{2}\right] \in H^{1}(M) \Rightarrow \mathcal{A}\left(L-\lambda_{1}\right)=\mathcal{A}\left(L-\lambda_{2}\right)
$$

It allows us to introduce the following notation : if $w \in H^{1}(M)$ is a cohomological class, $\mathcal{A}_{w}(L)=\mathcal{A}(L-\lambda)$ where $\lambda$ is any closed 1-form belonging to $w$. $A_{w}(L)$ is compact, non empty and invariant under $\left(f_{t}^{L}\right)$. Moreover, J. Mather proved in [17] that it is a Lipschitz graph above a part of the zero-section (see [10] or section 3 too).

As we are as interested in the Hamiltonian dynamics as in the Lagrangian ones, let us define the dual Aubry set :

- if $H$ is the Hamiltonian function associated to the Tonelli Lagrangian function $L$, its dual Aubry set is $\mathcal{A}^{*}(H)=\mathcal{L}_{L}(\mathcal{A}(L))$;

- if $w \in H^{1}(M)$ is a cohomological class, then $\mathcal{A}_{w}^{*}(H)=\mathcal{L}_{L}\left(\mathcal{A}_{w}(L)\right)$ is the $w$-dual Aubry set; let us notice that for any closed 1-form $\lambda$ belonging to $w$, we have : $T_{\lambda}\left(\mathcal{A}^{*}\left(H \circ T_{\lambda}\right)\right)=\mathcal{A}_{w}^{*}(H)$.

These sets are invariant under the Hamiltonian flow $\left(\Phi_{t}^{H}\right)$.

Another important invariant subset in the theory of Tonelli Lagrangian functions is the so-called Mather set. For it, there exists one definition (which is in [10], [15], [16]) : it is the closure of the union of the supports of the minimizing measures for $L$; it is denoted by $\mathcal{M}(L)$ and the dual Mather set is $\mathcal{M}^{*}(H)=\mathcal{L}_{L}(\mathcal{M}(L))$ which is compact, non empty and invariant under the flow $\left(\Phi_{t}^{H}\right)$. As for the Aubry set, if $w \in H^{1}(M)$ is a cohomological class, we define : $\mathcal{M}_{w}(L)=\mathcal{M}(L-\lambda)$ which is independent of the choice of the closed 1-form $\lambda$ belonging to $w$. Then $\mathcal{M}_{w}^{*}(H)=\mathcal{L}_{L}\left(\mathcal{M}_{w}(L)\right)=T_{\lambda}\left(\mathcal{M}^{*}\left(H \circ T_{\lambda}\right)\right)$ is invariant under $\left(\Phi_{t}^{H}\right)$; we name it the $w$-dual Mather set.

In a similar way, if $\mathcal{N}(L)$ is the Mañé set, the dual Mañé set is $\mathcal{N}^{*}(H)=\mathcal{L}_{L}(\mathcal{N}(L))$; we note that if $w \in H^{1}(M)$ and $\lambda \in w$, then $\mathcal{N}_{w}(L)=\mathcal{N}(L-\lambda)$ is independent of the choice of $\lambda \in w$ and then the $w$-dual Mañé set is $\mathcal{N}_{w}^{*}(H)=\mathcal{L}_{L}\left(\mathcal{N}_{w}(L)\right)=T_{\lambda}\left(\mathcal{N}^{*}(H \circ\right.$ $\left.T_{\lambda}\right)$ ); it is invariant under $\left(\Phi_{t}^{H}\right)$, compact and non empty but is not necessarily a graph.

For every cohomological class $w \in H^{1}(M)$, we have the inclusion : $\mathcal{M}_{w}^{*}(H) \subset$ $\mathcal{A}_{w}^{*}(H) \subset \mathcal{N}_{w}^{*}(H)$. Moreover, there exists a real number denoted by $\alpha_{H}(w)$ such that : $\mathcal{N}_{w}^{*}(H) \subset H^{-1}\left(\alpha_{H}(w)\right)$ (see [3] and [16]), i.e. each dual Mañé set is contained in an energy level. For $w=0$, the value $\alpha_{H}(0)$ is denoted by $c(L)$ and is named the "critical value" of $L$.

Definition. If $H: T^{*} M \rightarrow \mathbb{R}$ is a Tonelli Hamiltonian function, the tiered Aubry set, the tiered Mather set and the tiered Mañé set are :

$$
\mathcal{A}^{T}(L)=\bigcup_{w \in H^{1}(M)} \mathcal{A}_{w}(L) ; \quad \mathcal{M}^{T}(L)=\bigcup_{w \in H^{1}(M)} \mathcal{M}_{w}(L) ; \quad \mathcal{N}^{T}(L)=\bigcup_{w \in H^{1}(M)} \mathcal{N}_{w}(L) .
$$


Their dual sets are :

$$
\mathcal{A}_{*}^{T}(H)=\bigcup_{w \in H^{1}(M)} \mathcal{A}_{w}^{*}(H) ; \quad \mathcal{M}_{*}^{T}(H)=\bigcup_{w \in H^{1}(M)} \mathcal{M}_{w}^{*}(H) ; \quad \mathcal{N}_{*}^{T}(H)=\bigcup_{w \in H^{1}(M)} \mathcal{N}_{w}^{*}(H) .
$$

We shall prove in proposition 13 that the map $w \rightarrow \mathcal{N}_{w}^{*}(L)$ is upper semi-continuous (roughly speaking, these sets are "minimizing objects"), therefore $\mathcal{N}_{*}^{T}(H)$ is a closed subset of $T^{*} M$. It is unknown if such a result is true or false for the Aubry sets (see [18]). Concerning the topological structure of the tiered Mañé set, we have :

Proposition 1 Let $H: T^{*} M \rightarrow \mathbb{R}$ be a Tonelli Hamiltonian function. Then $\mathcal{N}_{*}^{T}(H)$ is closed, connected and if $\operatorname{dim} H^{1}(M) \geq 2$, for every $h \in \mathbb{R}$, the set $\mathcal{N}_{*}^{T}(H) \cap H^{-1}(h)$ is compact, connected and the restriction of $\left(\Phi_{t}^{H}\right)$ to $\mathcal{N}_{*}^{T}(H) \cap H^{-1}(h)$ is chain transitive.

Examples : 1) At first, let us consider the most simple completely integrable Hamiltonian function : $M=\mathbb{T}^{n}$ and $H(x, p)=\frac{1}{2}\|p\|^{2}$. In other words, we consider the geodesic flow on the flat torus. Then we have :

$$
\forall w \in H^{1}(M), \mathcal{M}_{w}^{*}(H)=\mathcal{A}_{w}^{*}(H)=\mathcal{N}_{w}^{*}(H)=\left\{(x, p) ; p=p_{0}\right\}
$$

where $p_{0}$ is a constant 1-form; i.e. each of these sets is an invariant Lagrangian torus, and all these sets fill up the phase space $T^{*} \mathbb{T}^{n}=\mathcal{A}_{*}^{T}(H)=\mathcal{M}_{*}^{T}(H)=\mathcal{N}_{*}^{T}(H)$.

2) If we perturb a completely integrable Hamiltonian system for the $C^{\infty}$ topology, we know that many invariant tori will persist (theorems K.A.M.) : they are dual Mather, Aubry and Mañé sets for certain cohomological classes. The weak K.A.M. theorems (see [10]) give an answer to the following question : what did happen to the invariant tori which disappeared during the perturbation? They prove the existence of positively invariant graphs above the zero section (which are not continuous, but in a certain sense Lagrangian), each of these graphs containing one dual Aubry set $\mathcal{A}_{c}^{*}(H)$ (which is invariant by the Hamiltonian flow $\left(\Phi_{t}^{H}\right)$ ) and possibly some pieces of the stable manifold of this Aubry set.

For the unperturbed system (completely integrable), we have shown that the Aubry sets fill up the phase space; but we shall prove that this situation si not generic (the definition of genericity is just after the theorem) :

Theorem 2 Let $H: T^{*} M \rightarrow \mathbb{R}$ be a $C^{\infty}$ generic Tonelli Hamiltonian function 2 . Then there exists a dense $G_{\delta}$ subset $G(H)$ of $\mathbb{R}$ such that for every $h \in G(H)$, then $\overline{\mathcal{A}_{*}^{T}(H)} \cap H^{-1}(h)$ has no interior in $H^{-1}(h)$; in particular, the interior of $\overline{\mathcal{A}_{*}^{T}(H)}$ is empty.

\footnotetext{
${ }^{2}$ Let us recall that we assume that $\operatorname{dim} M \geq 2$; the result is false if $\operatorname{dim} M=1$.
} 
In 1996, R. Mañé introduced the notion of "generic Lagrangian function" in [14]: "a certain property holds for a generic Lagrangian $L$ if, given a strictly convex and superlinear Lagrangian $L_{0}$, there exists a residual subset $\mathcal{O} \subset C^{\infty}(M)$ such that the given property holds for every Lagrangian $L$ of the form $L=L_{0}+\psi, \psi \in \mathcal{O}$ ".

Then we define (it is the dual definition for the Hamiltonian functions) :

Definition. A certain property holds for a generic Hamiltonian $H$ if, given a Tonelli Hamiltonian function $H_{0}$, there exists a residual subset $\mathcal{O} \subset C^{\infty}(M)$ such that the given property holds for every Hamiltonian $H$ of the form $H=H_{0}-\psi, \psi \in \mathcal{O}$.

The theorem 2 proves that in the sense of the Baire's category the tiered Aubry set is small (it is not true for the measure category when $M=\mathbb{T}^{n}$ : see the (strong) K.A.M. theorems). We may ask ourselves the same question for the Mañé set. Let us recall :

Definition. If $A$ is a closed set invariant under the flow $\left(\Phi_{t}^{H}\right)$, its stable manifold $W^{s}\left(A,\left(\Phi_{t}^{H}\right)\right)$ (resp. its unstable manifold $W^{u}\left(A,\left(\Phi_{t}^{H}\right)\right)$ ) is defined by :

$$
W^{s}\left(A,\left(\Phi_{t}^{H}\right)\right)=\left\{\xi \in T^{*} M ; \lim _{t \rightarrow+\infty} d\left(\Phi_{t}^{H}(\xi), A\right)=0\right\}
$$

(resp :

$$
\left.W^{u}\left(A,\left(\Phi_{t}^{H}\right)\right)=\left\{\xi \in T^{*} M ; \lim _{t \rightarrow-\infty} d\left(\Phi_{t}^{H}(\xi), A\right)=0\right\}\right) .
$$

Then it is known that $\mathcal{N}_{c}^{*}(H) \subset W^{s}\left(\mathcal{A}_{c}^{*}(H) ;\left(\Phi_{t}^{H}\right)\right) \cap W^{u}\left(\mathcal{A}_{c}^{*}(H) ;\left(\Phi_{t}^{H}\right)\right.$ ) (see [10] for example); we obtain :

Corollary 3 For $H: T^{*} M \rightarrow \mathbb{R}$ generic, the set $W^{s}\left(\overline{\mathcal{A}_{*}^{T}(H)} ;\left(\Phi_{t}^{H}\right)\right) \cup W^{u}\left(\overline{\mathcal{A}_{*}^{T}(H)} ;\left(\Phi_{t}^{H}\right)\right)$ has no interior; in particular, the tiered Mañé set $\mathcal{N}_{*}^{T}(H)$ has no interior.

REMARK. A usual default of the genericity results is that in general, one proves these results by using Baire's theorem but one cannot exhibit one single example of a "generic object". It is not the case of our result, and we obtain easily examples of such "generic Tonelli Hamiltonian functions". Let us consider a Hamiltonian function $H$ whose flow is Anosov on every regular energy level (for example the geodesic flow on a surface with negative curvature). Then the restriction of the Hamiltonian flow to every connected regular level is transitive; therefore, if $c$ is the critical value of $H$, the set $D$ of the points $p$ of $T^{*} M$ whose orbit is dense in their energy level $E_{p}$ above $c$ is a dense $G_{\delta}$ subset $G$ of $\{x \in T M ; H(x)>c\}$. But it is known that every orbit of a point of $\mathcal{N}_{*}^{T}(H)$ is a lipschitz graph above a part of the zero section, and therefore doesn't meet $G$ : its interior is then empty. 
Before proving theorem 2, we shall prove the two following results, the first one explaining in particular what happens in the "non generic case" (when the interior of $\overline{\mathcal{A}^{T}(L)}$ is non empty), the second one stating precisely which generic property we need :

Proposition 4 Let $L: T M \rightarrow \mathbb{R}$ be a Tonelli Lagrangian function; then the interior of $\overline{\mathcal{A}^{T}(L)}$ has a dense subset of periodic points with period in $\mathbb{N}^{*}$ whose orbits are graphs above a part of the zero section, and have no conjugate point.

Proposition 5 For the Tonelli Lagrangian functions, the following property is generic: "if $P$ is a periodic orbit which is a graph above a part of the zero section, which has no conjugate point and whose period is an integer $N \geq 1$, then :

- either $P$ is hyperbolic and isolated among the $N$-periodic orbits;

- or in every neighborhood of $P$ there exists an open subset of points whose orbit has conjugate points".

As we know that every orbit contained in $\overline{\mathcal{A}^{T}(L)}$ has no conjugate point (see section 2 for details), the last assertion of theorem 2 is an easy consequence of propositions 4 and 5. Then, we use a standard Baire argument to deduce the first assertion of theorem 2 (see the end of section 4 for the details).

Let us now mention and comment on some related results (in these results, the notion of genericity is not exactly the one which we defined before) :

1. in [13], M. Herman proved that for $C^{\infty}$ generic exact symplectic twist maps of the annulus $T^{*} \mathbb{T}^{1}$, there doesn't exist any invariant curve containing a periodic point (section I.5) : it implies that for such a generic twist map, the closure of the union of the Aubry-Mather sets has no interior;

2. in [12, M. Herman announced : for the $C^{\infty}$ generic exact symplectic diffeomorphisms $f$ of $T^{*} \mathbb{T}^{n}$ which are homotopic to the identity, if $I(f)$ is the closure of the union the invariant K.A.M. tori, then $I(f)$ has no interior;

3. I proved in [1] that the generic $C^{\infty}$ symplectic diffeomorphisms of any compact symplectic manifold verify : "the closure of the union of the periodic K.A.M. tori in equal to the closure of the set of the completely elliptic periodic points"; this implies of course that for such a generic symplectic diffeomorphism, the closure of the union of the K.A.M. tori has no interior.

We may ask ourselves the following question : when $M=\mathbb{T}^{n}$, is our set (the closure of $\mathcal{A}^{T}(L)$ ) different from the one introduced by M. Herman in [12]? Let us give a definition :

Definition. Let $H: T^{*} \mathbb{T}^{n} \rightarrow \mathbb{R}$ be a Tonelli hamiltonian function. A K.A.M. torus for $H$ is a Lagrangian $C^{\infty}$ graph $G$ above the zero section which is invariant by 
$\left(\Phi_{t}^{H}\right)$ and such that the restriction of the flow to $G$ is conjugated to an ergodic flow : $(\theta \rightarrow \theta+t \alpha)$.

The closure of the union of the K.A.M. tori for $H$ is denoted by $\mathcal{I}(H)$.

These K.A.M. tori are in fact Lipschitz graphs and on every compact, the Lipschitz constant may be chosen uniformly (it is only an adjustment of the results of [12]). Moreover, it is proved in [10] that every exact Lagrangian invariant $C^{1}$ graph is the graph of the derivative of a so called "weak K.A.M. solution", and therefore every Lagrangian invariant $C^{1}$ graph meets an Aubry set (for a certain cohomology class); if such a Lagrangian graph is a K.A.M. tori, it is in fact a Aubry set (because its dynamic are minimal). We deduce : $\mathcal{I}(H) \subset \mathcal{N}_{*}^{T}(H)$.

We shall build an example such that the tiered Aubry set is not in the closure of the union of the K.A.M. tori :

Proposition 6 There exists a $C^{\infty}$ Tonelli Lagrangian function $L: T \mathbb{T}^{2} \rightarrow \mathbb{R}$ and an open subset $\mathcal{U}$ of $C^{\infty}\left(\mathbb{T}^{2}\right)$ which contains 0 such that : for every $\psi \in \mathcal{U}$, there exists a periodic orbit $P$ for $\left(f_{t}^{L+\psi}\right)$ which belongs to $\mathcal{A}^{T}(L+\psi)$, but $\mathcal{L}_{L}(P)$ is not in the closure of the union of the K.A.M. tori for $H-\psi$ ( $H$ is the Hamiltonian function associated to $L$ ).

REMARK. This result is not very surprising; it corresponds to the existence of Birkhoff instability regions for twist maps : in these regions, there exist periodic orbits which are some Mather sets, but there exists no "K.A.M. curve".

\section{Peierls barrier, Mañé potential, Aubry and Mañé sets, proof of proposition 1}

We gather in this sections some well-known results; the ones concerning the Peierls barrier are essentially due to A. Fathi (see [10]), the others concerning Mañé potential are given in [14], [5] and 6].

At the end of this section, we prove some new results and proposition 1.

In the whole section, $L$ is a Tonelli Lagrangian function. At first, let us introduce some notations (we simplify the notation of the critical value : $c=c(L)$ ) :

Notations.

- given two points $x$ and $y$ in $M$ and $T>0$, we denote by $\mathcal{C}_{T}(x, y)$ the set of absolutely continuous curves $\gamma:[0, T] \rightarrow M$ with $\gamma(0)=x$ and $\gamma(T)=y$;

- the Lagrangian action along an absolutely continuous curve $\gamma:[a, b] \rightarrow M$ is defined by :

$$
A_{L}(\gamma)=\int_{a}^{b} L(\gamma(t), \dot{\gamma}(t)) d t
$$


- for each $t>0$, we define the function $h_{t}: M \times M \rightarrow \mathbb{R}$ by $: h_{t}(x, y)=$ $\inf \left\{A_{L+c}(\gamma) ; \gamma \in \mathcal{C}_{t}(x, y)\right\}$

- the Peierls barrier is then the function $h: M \times M \rightarrow \mathbb{R}$ defined by :

$$
h(x, y)=\liminf _{t \rightarrow+\infty} h_{t}(x, y)
$$

- we define the (Mañé) potential $m: M \times M \rightarrow \mathbb{R}$ by $: m(x, y)=\inf \left\{A_{L+c}(\gamma) ; \gamma \in\right.$ $\left.\bigcup_{T>0} \mathcal{C}_{T}(x, y)\right\}=\inf \left\{h_{t}(x, y) ; t>0\right\}$.

Then, the Mañé potential verifies :

Proposition 7 We have :

1. $m$ is finite and $m \leq h$;

2. $\forall x, y, z \in M, m(x, z) \leq m(x, y)+m(y, z)$;

3. $\forall x \in M, m(x, x)=0$;

4. if $x, y \in M$, then $m(x, y)+m(y, x) \geq 0$;

5. if $M_{1}=\sup \{L(x, v) ;\|v\| \leq 1\}$, then $: \forall x, y \in M,|m(x, y)| \leq\left(M_{1}+c\right) d(x, y)$;

6. $m: M \times M \rightarrow \mathbb{R}$ is $\left(M_{1}+c\right)$-Lipschitz.

Now we can define :

DEFINITION.

- a absolutely continuous curve $\gamma: I \rightarrow M$ defined on an interval $I$ is a ray if :

$$
\forall[a, b] \subset I, A_{L+c}\left(\gamma_{\mid[a, b]}\right)=h_{(b-a)}(\gamma(a), \gamma(b)) ;
$$

a ray is always a solution of the Euler-Lagrange equations;

- a absolutely continuous curve $\gamma: I \rightarrow M$ defined on an interval $I$ is semistatic if :

$$
\forall[a, b] \subset I, m_{c}(\gamma(a), \gamma(b))=A_{L+c}\left(\gamma_{\mid[a, b]}\right) ;
$$

a semistatic curve is always a ray;

- the Mañé set is then : $\mathcal{N}(L)=\left\{v \in T M ; \gamma_{v}\right.$ is semistatic $\}$ where $\gamma_{v}$ designates the solution $\gamma_{v}: \mathbb{R} \rightarrow M$ of the Euler-Lagrange equations with initial condition $v$ for $t=0 ; \mathcal{N}(L)$ is contained in the critical energy level;

- a absolutely continuous curve $\gamma: I \rightarrow M$ defined on an interval $I$ is static if :

$$
\forall[a, b] \subset I,-m_{c}(\gamma(b), \gamma(a))=A_{L+c}\left(\gamma_{\mid[a, b]}\right) ;
$$

a static curve is always a semistatic curve;

- the Aubry set is then : $\mathcal{A}(L)=\left\{v \in T M ; \gamma_{v}\right.$ is static $\}$. 
The following result is proved in [6] :

Proposition 8 If $v \in T M$ is such that $\gamma_{v \mid[a, b]}$ is static for some $a<b$, then $\gamma_{v}: \mathbb{R} \rightarrow$ $M$ is static, i.e. $v \in \mathcal{A}(L)$.

The Peierls barrier verifies (this proposition contains some results of [9], [10] and [4]) :

Proposition 9 (properties of the Peierls barrier $h$ )

1. the values of the map $h$ are finite and $m \leq h$;

2. if $M_{1}=\sup \{L(x, v) ;\|v\| \leq 1\}$, then:

$$
\forall x, y, x^{\prime}, y^{\prime} \in M,\left|h(x, y)-h\left(x^{\prime}, y^{\prime}\right)\right| \leq\left(M_{1}+c\right)\left(d\left(x, x^{\prime}\right)+d\left(y, y^{\prime}\right)\right)
$$

therefore $h$ is Lipschitz;

3. if $x, y \in M$, then $h(x, y)+h(y, x) \geq 0$; we deduce $: \forall x \in M, h(x, x) \geq 0$;

4. $\forall x, y, z \in M, h(x, z) \leq h(x, y)+h(y, z)$;

5. $\forall x \in M, \forall y \in \pi(\mathcal{A}(L)), m(x, y)=h(x, y) \quad$ and $m(y, x)=h(y, x)$;

6. $\forall x \in M, h(x, x)=0 \Longleftrightarrow x \in \pi(\mathcal{A}(L))$.

The last item of this proposition gives us a characterization of the projected Aubry set $\pi(\mathcal{A}(L))$. Moreover, we have :

Proposition 10 (A. Fathi, [10], 6.3.3) When t tends to $+\infty$, uniformly on $M \times M$, the function $h_{t}$ tends to the Peierls barrier $h$.

A corollary of this result is given in [6] :

Corollary 11 ([6], 4-10.9) All the rays defined on $\mathbb{R}$ are semistatic.

Let us give some properties of the Aubry and Mañé sets (see [15] and [5]) :

Proposition 12 Let $L: T M \rightarrow \mathbb{R}$ be a Tonelli lagrangian function. Then :

- the Aubry and Mañé set are compact, non empty and $\mathcal{A}(L) \subset \mathcal{N}(L)$;

- the Aubry set is a Lipschitz graph above a part of the zero section;

- if $\gamma: \mathbb{R} \rightarrow M$ is semistatic, then $(\gamma, \dot{\gamma})$ is a Lipschitz graph above a part of the zero section;

- the $\omega$ and $\alpha$-limit sets of every point of the Mañé set are contained in the Aubry set. 
We denote by $\Lambda^{1}(M)$ the set of $\left(C^{\infty}\right)$ closed 1-forms of $M$ and $\mathcal{K}(T M)$ the set of non empty compact subset of $T M$. Let us now prove :

Proposition 13 Let $L: T M \rightarrow \mathbb{R}$ be a Tonelli Lagrangian function. The map $K:(\psi, \lambda) \in C^{\infty}(M) \times \Lambda^{1}(M) \rightarrow \mathcal{N}(L+\psi+\lambda) \in \mathcal{K}(T M)$ is upper semi-continuous.

Proof of PROPOSITION 13 :

Let $H: T^{*} M \rightarrow \mathbb{R}$ be the Tonelli Hamiltonian function associated to $L$. we prove :

Lemma 14 The map $(\psi, \lambda) \in C^{\infty}(M) \times \Lambda^{1}(M) \rightarrow \alpha_{H-\psi}(\lambda)=c(L+\psi-\lambda)$ is continuous.

Proof of LEMma 14: We use the characterization of the critical value with the holonomic (probability) measures (see [15] or [6] for the exact definition of holonomic measure) : $-c(L)$ is the minimum of $A_{L}(\mu)$ among the holonomic measures $\mu$; then each such minimizing measures is invariant under $\left(f_{t}^{L}\right)$ and is contained in the energy level $\mathcal{L}_{L}^{-1}\left(H^{-1}(c(L))\right)$.

To prove that $(\psi, \lambda) \rightarrow \alpha_{H-\psi}(\lambda)=c(L+\psi-\lambda)$ is continuous, we only need to prove the continuity at $(0,0)$.

As $L$ is superlinear, there exists a compact $K \subset T M$ and a neighborhood $\mathcal{V}$ of $(0,0)$ in $C^{\infty}(M) \times \Lambda^{1}(M)$ such that : for every $(\psi, \lambda) \in \mathcal{V}$, for every holonomic measure $\mu$ such that the support of $\mu$ meets $T M \backslash K, \mu$ is not minimizing for $L+\psi-\lambda$. Indeed, let us fix $\mu_{0}$ any holonomic measure on $T M$; there exists a neighborhood $\mathcal{V}_{0}$ of $(0,0)$ in $C^{\infty}(M) \times \Lambda^{1}(M)$ and a constant $\ell \in \mathbb{R}$ such that $: \forall(\psi, \lambda) \in \mathcal{V}_{0}, A_{L+\psi-\lambda}\left(\mu_{0}\right) \leq$ $\ell$. Because $L$ is superlinear, there exists a constant $C_{1} \in \mathbb{R}$ such that : $\forall(\psi, \lambda) \in$ $\mathcal{V}_{0}, \forall(x, v) \in T M,(L+\psi-\lambda)(x, v) \leq \ell \Rightarrow\|v\| \leq C_{1}$. The Hamiltonian function $H$ associated to $L$ being superlinear too, there exists a constant $C \in \mathbb{R}$ such that : if $(\psi, \lambda) \in \mathcal{V}_{0}$, if $(x, v)$ and $\left(x_{0}, v_{0}\right)$ are in the same energy level for $L+\psi-\lambda$ and if $\left\|v_{0}\right\| \leq C_{1}$, then $\|v\| \leq C$. Hence, if $(\psi, \lambda) \in \mathcal{V}_{0}$, if $\mu_{L+\psi-\lambda}$ is a minimizing measure for $L+\psi-\lambda$, we have : $A_{L+\psi-\lambda}\left(\mu_{L+\psi-\lambda}\right)=-c(L+\psi-\lambda) \leq \ell$. It implies that there exists $(x, v) \in \operatorname{supp}\left(\mu_{L+\psi-\lambda}\right)$ such that $(L+\psi-\lambda)(x, v) \leq \ell$ and then $\|v\| \leq C_{1}$. But, $\mu_{L+\psi-\lambda}$ being minimizing, every point of its support has the same energy as $(x, v)$ and then : $\forall(X, V) \in \operatorname{supp}\left(\mu_{L+\psi-\lambda}\right),\|V\| \leq C$. We choose $K=\{(x, v) ;\|v\| \leq C\}$.

We have then to minimize a continuous function $\mu \rightarrow A_{L+\psi-\lambda}(\mu)$ on a compact set (the set of holonomic probabilities with support in $K$ ), we know that the minimum depends continuously on $(\psi, \lambda)$.

From lemma 14 and the fact that $\mathcal{N}(L) \subset \mathcal{L}^{-1}\left(H^{-1}(c(L))\right)$, we deduce that the Mañé set cannot "explode" : for every $(\psi, \lambda) \in C^{\infty}(M) \times \Lambda^{1}(M)$, there exists a neighborhood $\mathcal{V}$ of $(\psi, \lambda)$ and a compact $K$ of $T M$ such that : $\forall\left(\psi^{\prime}, \lambda^{\prime}\right) \in \mathcal{V}, \mathcal{N}(L+$ $\left.\psi^{\prime}-\lambda^{\prime}\right) \subset K$. 
Let us assume that the proposition 13 is not true. Then there exists a sequence $\left(\psi_{n}, \lambda_{n}\right)$ in $C^{\infty}(M) \times \Lambda^{1}(M)$ which converges to $(\psi, \lambda)$ and a sequence $\left(x_{n}, v_{n}\right) \in T M$ converging to $(x, v)$ such that :

- $\forall n,\left(x_{n}, v_{n}\right) \in \mathcal{N}\left(L+\psi_{n}+\lambda_{n}\right)$;

- $(x, v) \notin \mathcal{N}(L+\psi+\lambda)$.

As $(x, v) \notin \mathcal{N}(L+\psi+\lambda)$, the arc $\left(t \rightarrow \gamma(t)=\pi \circ f_{t}^{L+\psi+\lambda}(x, v)\right)$ is not a ray for the Lagrangian $L+\psi+\lambda$ and there exists $[a, b] \subset \mathbb{R}$ and $\varepsilon>0$ such that :

$$
A_{L+\psi+\lambda}\left(\gamma_{\mid[a, b]}\right) \geq h_{(b-a)}^{L+\psi+\lambda}(\gamma(a), \gamma(b))-(b-a) c(L+\psi+\lambda)+\varepsilon .
$$

When $n$ tends to the infinite, if we define $\gamma_{n}(t)=\pi \circ f_{t}^{L+\psi_{n}+\lambda_{n}}\left(x_{n}, v_{n}\right)$, then $\left(\gamma_{n}, \dot{\gamma}_{n}\right)$ converges uniformly on any compact interval to $(\gamma, \dot{\gamma})$.

We deduce that for $n$ big enough, we have :

$$
A_{L+\psi_{n}+\lambda_{n}}\left(\gamma_{n \mid[a, b]}\right) \geq h_{(b-a)}^{L+\psi_{n}+\lambda_{n}}\left(\gamma_{n}(a), \gamma_{n}(b)\right)-c\left(L+\psi_{n}+\lambda_{n}\right)+\frac{\varepsilon}{2}
$$

therefore $\gamma_{n}$ is not a ray, and then is not semistatic. It is a contradiction.

We deduce a part of proposition 1 :

Corollary 15 The tiered Mañé set $\mathcal{N}_{*}^{T}(H)=\bigcup_{c \in H^{1}(M)} \mathcal{N}_{c}^{*}(H)$ is closed.

Corollary 16 Let $L: T M \rightarrow \mathbb{R}$ be a Tonelli Lagrangian function and let $(x, v) \in T M$ be such that $(x, v) \notin \mathcal{N}^{T}(L)$. Then there exist :

- an open neighborhood $U$ of $(x, v)$ in $T M$;

- an open neighborhood $\mathcal{U}$ of 0 in $C^{\infty}(M)$;

such that:

$$
\forall \psi \in \mathcal{U}, U \cap \mathcal{N}^{T}(L+\psi)=\emptyset .
$$

Proof of COROLlary [16]: We know that $(x, v) \notin \mathcal{N}^{T}(L)$ and that $\mathcal{N}^{T}(L)$ is closed; thus there exists a compact neighborhood $K$ of $(x, v)$ in $T M$ such that $K \cap \mathcal{N}^{T}(L)=\emptyset$. Let $H$ be the Tonelli Hamiltonian function associated to $L$. Then J. Mather proved (see [16] or [6]) that $\alpha_{H}$ is convex and superlinear. Therefore there exists a convex compact subset $C$ of $H^{1}(M)$ and a real $R>0$ such that :

- $\forall\left(x_{1}, v_{1}\right) \in K, H\left(\mathcal{L}_{L}\left(x_{1}, v_{1}\right)\right)<\frac{R}{2}$;

- $\forall w \in \partial C, \alpha_{H}(w)>R$. 
We deduce from the proposition 13 that if we define : $\mathcal{N}_{\partial C}(L)=\bigcup_{w \in \partial C} \mathcal{N}_{w}(L)$, then the map $\psi \in C^{\infty}(M) \rightarrow \mathcal{N}_{\partial C}(L+\psi)$ is upper semi-continuous. Therefore, the map $\psi \in C^{\infty}(M) \rightarrow H \circ \mathcal{L}_{L}\left(\mathcal{N}_{\partial C}(L+\psi)\right)=\alpha_{H-\psi}(\partial C)$ is upper semi-continuous too. We have :

- $\left.\alpha_{H}(\partial C) \subset\right] R,+\infty[$;

- the map $\psi \in C^{\infty}(M) \rightarrow \alpha_{H-\psi}(\partial C)$ is upper semi-continuous.

Then, there exists a neighborhood $\mathcal{U}$ of 0 in $C^{\infty}(M)$ such that : $\forall \psi \in U, \alpha_{H-\psi}(\partial C) \subset$ ]$R,+\infty\left[\right.$. Moreover, if $\mathcal{U}$ is small enough, we have $\left.: \forall \psi \in \mathcal{U},(H-\psi) \circ \mathcal{L}_{L}(K) \subset\right]-\infty, \frac{R}{2}[$. These two facts implies that :

- $\forall \psi \in U, \forall w \in H^{1}(M) \backslash C, \alpha_{H-\psi}(w)>R$;

- $\forall \psi \in U, \forall\left(x_{1}, v_{1}\right) \in K,(H-\psi)\left(\mathcal{L}_{L}(x, v)\right)<\frac{R}{2}$.

Then, if $\psi \in \mathcal{U}$, for every $w \in H^{1}(M) \backslash C$, for every $\left(x_{1}, v_{1}\right) \in K:\left(x_{1}, v_{1}\right) \notin \mathcal{N}_{w}(L+\psi)$. Moreover, the map $\psi \in C^{\infty}(M) \rightarrow \mathcal{N}_{C}(L+\psi)=\bigcup_{w \in C} \mathcal{N}_{w}(L)$ is upper semi-continuous (proposition 13). There exists a neighborhood $\mathcal{V}$ of 0 in $C^{\infty}(M)$ such that : $\forall \psi \in$ $\mathcal{V}, \mathcal{N}_{C}(L+\psi) \cap K=\emptyset$.

We obtain the conclusion of the corollary with $\mathcal{U} \cap \mathcal{V}$ and the interior of $K$.

End of The PRoOf of PRoposition 1 : Let $L$ be the Tonelli Lagrangian function associated to $H$. We have proved in proposition 13 that the map $w \in H^{1}(M) \rightarrow$ $\mathcal{N}_{w}(L) \in \mathcal{K}(T M)$ is upper semi-continuous. Moreover, we know that each Mañé set $\mathcal{N}_{w}(L)$ is connected (and chain transitive). We deduce :

Lemma 17 For every arcwise connected subset $C$ of $H^{1}(M)$, the restriction of $\left(f_{t}^{L}\right)$ to $\bigcup_{w \in C} \mathcal{N}_{w}(L)$ is chain transitive $\left(\bigcup_{w \in C} \mathcal{N}_{w}(L)\right.$ is therefore connected when $C$ is closed).

Proof of Lemma 17: Let $x, y$ be two points of $\bigcup_{w \in C} \mathcal{N}_{w}(L)$ and $T>0, \varepsilon>0$; we want to connect $x$ to $y$ via a $(\varepsilon, T)$-chain. Let $w_{1}, w_{2} \in C$ be such that $x \in \mathcal{N}_{w_{1}}(L)$ and $y \in \mathcal{N}_{w_{2}}(L)$. The set $C$ being arcwise connected, there exists a continuous arc $w:[0,1] \rightarrow C$ such that $w(0)=w_{1}$ and $w(1)=w_{2}$. The map $t \in[0,1] \rightarrow \mathcal{N}_{w(t)}(L)$ is then upper semi-continuous. Therefore for every $t_{0} \in[0,1]$, there exists $\alpha\left(t_{0}\right)>0$ such that : $\forall t \in[0,1] \cap] t_{0}-\alpha\left(t_{0}\right), t_{0}+\alpha\left(t_{0}\right)\left[, \rho\left(\mathcal{N}_{w\left(t_{0}\right)}(L), \mathcal{N}_{w(t)}(L)\right)<\frac{\varepsilon}{3}\right.$ where we define :

$$
\rho(A, B)=\sup \{d(b, A) ; b \in B\} .
$$

We deduce : $\forall t \in[0,1] \cap] t_{0}-\alpha\left(t_{0}\right), t_{0}+\alpha\left(t_{0}\right)\left[, d\left(\mathcal{N}_{w\left(t_{0}\right)}(L), \mathcal{N}_{w(t)}(L)\right)<\frac{\varepsilon}{3}\right.$ where, if $A, B \subset T M$, we define : $d(A, B)=\inf _{a \in A, b \in B} d(a, b)$. 
$[0,1]$ being compact, using a finite covering, we find a finite sequence $t_{0}=0<\cdots<$ $t_{N}=1$ such that : $\forall j, d\left(\mathcal{N}_{w\left(t_{j}\right)}, \mathcal{N}_{w\left(t_{j+1}\right)}\right)<\frac{\varepsilon}{3}$. Then we define a (finite) sequence of points :

- $x_{0}=x ; x_{2 N+1}=y$;

- for every $j \in\{0, \ldots, N\}, x_{2 j}, x_{2 j+1} \in \mathcal{N}_{w\left(t_{j}\right)}(L)$;

- for every $j \in\{1, \ldots, N\}, d\left(x_{2 j-1}, x_{2 j}\right)<\frac{\varepsilon}{3}$.

Every $x_{j}$ being in the chain recurrent set of $\bigcup_{w \in C} \mathcal{N}_{w}(L)$ and each $x_{2 j}$ being connected to $x_{2 j+1}$ by a $\left(\frac{\varepsilon}{3}, T\right)$ chain of $\bigcup_{w \in C} \mathcal{N}_{w}(L)$, we obtain easily a chain passing through $x, x_{1}, \ldots, x_{2 N+1}=y$.

Using lemma 17 for $C=H^{1}(M)$, we deduce that $\mathcal{N}_{*}^{T}(H)$ is chain transitive and therefore connected.

To deduce the end of the proof of proposition 1, we assume that $\operatorname{dim} H^{1}(M) \geq 2$; in this case, we notice that if $h \in \mathbb{R}, \alpha_{H}^{-1}(h)$ is arcwise connected (it is either a convex subset of $H^{1}(M)$ or the boundary of a compact convex subset of $H^{1}(M)$ whose dimension is at least 2 , which is homeomorphic to a connected sphere) and closed. Moreover, we have :

$$
\mathcal{N}_{*}^{T}(H) \cap H^{-1}(h)=\bigcup_{w \in \alpha_{H}^{-1}(h)} \mathcal{N}_{w}^{*}(L) .
$$

\section{Radially transformed set and Aubry set, proof of proposition 4}

Definition. Let $T>0$; we define :

- the set $\mathcal{R}_{T}(L)$ of the $T$-radially transformed points under $\left(f_{t}^{L}\right)$ is :

$$
\mathcal{R}_{T}(L)=\left\{(x, v) \in T M ; \pi\left(f_{T}^{L}(x, v)\right)=x\right\} ;
$$

its dual set is then $\mathcal{R}_{T}^{*}(H)=\mathcal{L}\left(\mathcal{R}_{T}(L)\right)$;

- the set $\mathcal{P}_{T}(L)$ is the set of the $T$-periodic orbits of the Lagrangian flow $\left(f_{t}^{L}\right)$ :

$$
\mathcal{P}_{T}(L)=\left\{(x, v) \in T M ; f_{T}^{L}(x, v)=(x, v)\right\} ;
$$

its dual set is then $\mathcal{P}_{T}^{*}(H)=\mathcal{L}\left(\mathcal{P}_{T}(L)\right)$. 
We note that $\mathcal{P}_{T}(L) \subset \mathcal{R}_{T}(L)$ and that if $\lambda$ is a $C^{\infty}$ closed 1-form, we have : $\mathcal{P}_{T}(L-\lambda)=\mathcal{P}_{T}(L), \mathcal{R}_{T}(L-\lambda)=\mathcal{R}_{T}(L)$.

Some of the radially transformed points which we described before are minimizing in a certain sense :

Proposition 18 (and definition) Let $\lambda$ be a closed $C^{\infty}$ 1-form of $M$. Then for every $x \in M$, the set :

$$
\Gamma_{T}(L, \lambda ; x)=\left\{\gamma \in \mathcal{C}_{T}(x, x) ; \forall \eta \in \mathcal{C}_{T}(x, x), A_{L-\lambda}(\gamma) \leq A_{L-\lambda}(\eta)\right\}
$$

is non empty and each $\gamma \in \Gamma_{T}(L, \lambda ; x)$ is a solution of the Euler-Lagrange equations. Moreover, if $\mu$ is a closed 1-form such that $[\mu]=[\lambda]$, then $\Gamma_{T}(L, \lambda ; x)=\Gamma_{T}(L, \mu ; x)$. This allows us to define for every $w \in H^{1}(M): \Gamma_{T}(L, w ; x)=\Gamma_{T}(L, \lambda ; x)$ if $[\lambda]=w$ and :

- $\mathcal{R}_{T}(L, w ; x)=\left\{(\gamma(0), \dot{\gamma}(0)) ; \gamma \in \Gamma_{T}(L, w ; x)\right\}$;

- $\mathcal{R}_{T}(L, w)=\bigcup_{x \in M} \mathcal{R}_{T}(L, w ; x)$;

the sets $\mathcal{R}_{T}(L, w ; x)$ and $\mathcal{R}_{T}(L, w)$ are closed and we have $: \mathcal{R}_{T}(L, w) \subset \mathcal{R}_{T}(L)$.

This proposition is an easy consequence of Tonelli theorem (see [10]).

Let us explain how the radially transformed minimizing points allow us to approximate the Aubry set :

Proposition 19 Let $w \in H^{1}(M), \varepsilon>0$ and let $L: T M \rightarrow \mathbb{R}$ be a Tonelli Lagrangian function. Then there exists $T_{0}>0$ such that:

$$
\forall T \geq T_{0}, \forall(x, v) \in \mathcal{R}_{T}(L, w), x \in \pi\left(\mathcal{A}_{w}(L)\right) \Longrightarrow d\left((x, v), \mathcal{A}_{w}(L)\right) \leq \varepsilon .
$$

What this last proposition says is : the family $\left(\mathcal{R}_{T}(L, w) \cap \pi^{-1}\left(\mathcal{A}_{w}(L)\right)\right)_{T>0}$ of nonempty compact subsets of $T M$ tends to $\mathcal{A}_{w}(L)$ (for the Hausdorff topology) when $T$ tends to $+\infty$. This will be one of the main ingredients of our proof of theorem 2 , which will give us some points near the Aubry set but not in the Aubry set (for generic Lagrangian functions).

Proof of PRoposition 19: Let us assume that the result is not true; then we may find a sequence $\left(T_{n}\right)_{n \in \mathbb{N}}$ in $\mathbb{R}_{+}^{*}$ tending to $+\infty$, a sequence $\left(x_{n}, v_{n}\right)$ of points of $\mathcal{R}_{T_{n}}(L, w) \cap \pi^{-1}\left(\mathcal{A}_{w}(L)\right)$ such that $: \forall n \in \mathbb{N}, d\left(\left(x_{n}, v_{n}\right), \mathcal{A}_{w}(L)\right) \geq \varepsilon$.

Now we use proposition [10: let $\lambda$ be a closed 1-form such that $[\lambda]=w$; then we know that if we define $h_{t}^{\lambda}: M \times M \rightarrow \mathbb{R}$ by $h_{t}^{\lambda}(x, y)=\inf \left\{A_{L-\lambda+\alpha_{H}(w)}(\gamma) ; \gamma \in \mathcal{C}_{t}(x, y)\right\}$ and $h^{\lambda}(x, y)=\liminf _{t \rightarrow+\infty} h_{t}^{\lambda}(x, y)$, the functions $h_{t}^{\lambda}$ tend uniformly to $h^{\lambda}$ when $t$ tends to $+\infty$; moreover, we know that $h^{\lambda}$ is Lipschitz and zero at every $(x, x)$ with $x \in \mathcal{A}_{w}(L)$. If $\gamma_{n}$ designates the solution of the Euler-Lagrange equations with initial condition 
$\left(\gamma_{n}(0), \dot{\gamma}_{n}(0)\right)=\left(x_{n}, v_{n}\right)$ we have then $: h_{T_{n}}^{\lambda}\left(x_{n}, x_{n}\right)=A_{L-\lambda+\alpha_{H}(w)}\left(\gamma_{n}\right)$ tends to 0 when $n$ tends to the infinite.

The sequence $\left(x_{n}, v_{n}\right)$ is bounded (it is a consequence of the so-called "a priori compactness lemma" (see [10], corollary 4.3.2)); therefore we may extract a converging subsequence : we call it $\left(x_{n}, v_{n}\right)$ again and $\left(x_{\infty}, y_{\infty}\right)$ is its limit. Let us notice that $x_{\infty} \in \pi\left(\mathcal{A}_{w}(L)\right)$ because $\mathcal{A}_{w}(L)$ is compact. Moreover, we have : $d\left(\left(x_{\infty}, v_{\infty}\right), \mathcal{A}_{w}(L)\right) \geq$ $\varepsilon$.

Let $\gamma_{\infty}$ be the solution of the Euler-lagrange equations such that $\left(\gamma_{\infty}(0), \dot{\gamma}_{\infty}(0)\right)=$ $\left(x_{\infty}, v_{\infty}\right)$. We want to prove that $\gamma_{\infty}$ is static : we shall obtain a contradiction. When $n$ is big enough, $\gamma_{n}\left(T_{n}\right)=\gamma_{n}(0)$ is close to $\gamma_{\infty}(0)$ and $\gamma_{n}(1)$ is close to $\gamma_{\infty}(1)$. Let us fix $\eta>0$; then we define $\Gamma_{n}^{\eta}:\left[0, T_{n}+2 \eta\right] \rightarrow M$ by :

- $\Gamma_{n \mid[0,1]}^{\eta}=\gamma_{\infty \mid[0,1]}$;

- $\Gamma_{n \mid[1,1+\eta]}^{\eta}$ is a short geodesic joining $\gamma_{\infty}(1)$ to $\gamma_{n}(1)$;

- $\forall t \in\left[1+\eta, T_{n}+\eta\right], \Gamma_{n}^{\eta}(t)=\gamma_{n}(t-\eta)$;

- $\Gamma_{n \mid\left[T_{n}+\eta, T_{n}+2 \eta\right]}^{\eta}$ is a short geodesic joining $\gamma_{n}\left(T_{n}\right)$ to $\gamma_{\infty}(0)$.

If we choose carefully a sequence $\left(\eta_{n}\right)$ tending to 0 , we have :

$$
\lim _{n \rightarrow \infty} A_{L-\lambda+\alpha_{H}(w)}\left(\Gamma_{n}^{\eta_{n}}\right)=\lim _{n \rightarrow \infty} A_{L-\lambda+\alpha_{H}(w)}\left(\gamma_{n}\right)=0 .
$$

Because the contribution to the action of the two small geodesic arcs tends to zero (if the $\eta_{n}$ are well chosen), this implies :

$$
A_{L-\lambda+\alpha_{H}(w)}\left(\gamma_{\infty \mid[0,1]}\right)+m^{\lambda}\left(\gamma_{\infty}(1), \gamma_{\infty}(0)\right) \leq 0
$$

where $m^{\lambda}$ designates Mañé potential for the Lagrangian function $L-\lambda$. We deduce then from the definition of Mañé potential that $m^{\lambda}\left(\gamma_{\infty}(0), \gamma_{\infty}(1)\right)+m^{\lambda}\left(\gamma_{\infty}(1), \gamma_{\infty}(0)\right)=0$ and that : $A_{L-\lambda+\alpha_{H}(w)}\left(\gamma_{\mid[0,1]}\right)=m^{\lambda}\left(\gamma_{\infty}(0), \gamma_{\infty}(1)\right)$. It implies then that $A_{L-\lambda+\alpha_{H}(w)}\left(\gamma_{\mid[0,1]}\right)=$ $-m^{\lambda}\left(\gamma_{\infty}(1), \gamma_{\infty}(0)\right)$. Let us notice that, changing slightly $\Gamma_{n}^{\eta}$, we obtain too :

$$
\forall[a, b] \subset\left[0,+\infty\left[, A_{L-\lambda+\alpha_{H}(w)}\left(\gamma_{\mid[a, b]}\right)=-m^{\lambda}\left(\gamma_{\infty}(b), \gamma_{\infty}(a)\right) ;\right.\right.
$$

therefore $\gamma_{\mid[0,+\infty[}$ is static. To conclude, we use the proposition 8 .

To finish this section we give a result which explains why in general the radially transformed points are not in a Mañé set : in this case, they would be periodic.

Proposition 20 Let $L$ be a Tonelli Lagrangian function and let $w \in H^{1}(M)$ be a cohomology class; then, for every $T>0$, we have :

$$
\mathcal{N}_{w}(L) \cap \mathcal{R}_{T}(L) \subset \mathcal{P}_{T}(L) \cap \mathcal{R}_{T}(L, w) \cap \mathcal{A}_{w}(L) .
$$


Proof of Proposition 20: By proposition 12, we know that if $(x, v) \in \mathcal{N}_{w}(L)$, then its orbit is a Lipschitz graph above a part of the zero section. Therefore, if $(x, v) \in \mathcal{N}_{w}(L) \cap \mathcal{R}_{T}(L)$, then the orbit of $(x, v)$ under $\left(f_{t}^{L}\right)$ is a graph (above a part of the zero section); as $\pi\left(f_{T}(x, v)\right)=\pi(x, v)$, we deduce that : $f_{T}(x, v)=(x, v)$ : $(x, v)$ is $T$-periodic for $\left(f_{t}\right)$, i.e. $(x, v) \in \mathcal{P}_{T}(L)$. Moreover, $\gamma_{v}$ is a ray : therefore it is minimizing between $\gamma_{v}(0)$ and $\gamma_{v}(T):(x, v) \in \mathcal{R}_{T}(L, w)$.

We deduce from from proposition 12 that every periodic orbit contained in $\mathcal{N}_{w}(L)$ is in $\mathcal{A}_{w}(L)$. Hence $(x, v) \in \mathcal{A}_{w}(L)$.

Corollary 21 Let $L$ be a Tonelli lagrangian function. Let $(x, v) \in T M$ be such that : $(x, v) \in \mathcal{R}_{T}(L) \backslash \mathcal{P}_{T}(L)$ for some $T>0$. Then there exist :

- an open neighborhood $U$ of $(x, v)$ in $T M$;

- an open neighborhood $\mathcal{U}$ of 0 in $C^{\infty}(M)$;

such that:

$$
\forall \psi \in \mathcal{U}, U \cap \mathcal{N}^{T}(L+\psi)=\emptyset
$$

This result is an easy consequence of proposition 20 and corollary 16 .

Let us now prove :

Proposition 22 Let $U$ be a non empty open subset of of $T M$ and $L: T M \rightarrow \mathbb{R}$ be a Tonelli Lagrangian function. Then :

- either there exists a non empty subset $U^{\prime} \subset U$ such that $U^{\prime} \cap \mathcal{A}^{T}(L)=\emptyset$;

- or there exists $N \in \mathbb{N}^{*}$ and a sequence $\left(x_{n}, v_{n}\right)$ of different $N$-periodic points contained in $\mathcal{A}^{T}(L) \cap U$ such that $\lim _{n \rightarrow \infty}\left(x_{n}, v_{n}\right)=\left(x_{0}, v_{0}\right)$; moreover the orbit of every $\left(x_{n}, v_{n}\right)$ is a graph above a part or the zero section, and has no conjugate point.

Of course, we deduce proposition 4 from this lemma : if $U \subset \overline{\mathcal{A}^{T}(L)}$ is a non empty open subset, we have found $(x, v) \in U$ which is periodic, whose orbit is a graph above a part or the zero section, and has no conjugate point.

Proof of PROPOSITION 22:

Let us consider a Tonelli Lagrangian function $L$ and let $U$ be a non empty subset of $T M$. There are two cases :

1. either $U \cap \mathcal{A}^{T}(L)=\emptyset$ : we have the first conclusion;

2. or there exists $(x, v) \in U \cap \mathcal{A}_{w}(L)$ for a certain $w \in H^{1}(M)$.

Let us choose $\alpha>0$ such that $\bar{B}((x, v), \alpha) \subset U$. We know that $\mathcal{A}_{w}(L)$ is a compact graph above a part of the zero section such that : $T_{x} M \cap \mathcal{A}_{w}(L)=\{(x, v)\}$. 
Therefore, there exists $\varepsilon>0$ such that $\forall\left(x, v_{1}\right) \in T_{x} M, d\left(\left(x, v_{1}\right), \mathcal{A}_{w}(L)\right)<\alpha \Rightarrow$ $d\left(\left(x, v_{1}\right),(x, v)\right)<\varepsilon$. By proposition 19, there exists $N_{0}>0$ such that :

$$
\forall N \geq N_{0}, \forall\left(x, v_{1}\right) \in \mathcal{R}_{N}(L, w, ; x), d\left(\left(x, v_{1}\right), \mathcal{A}_{w}(L)\right) \leq \alpha .
$$

We deduce that if $N \geq N_{0}: \mathcal{R}_{N}(L, w ; x) \subset U$.

Let us recall that the set $\mathcal{R}_{N}(L, w)=\bigcup_{y \in M} \mathcal{R}_{N}(L, w ; y)$ is a closed subset of $T M$ (proposition 18). Thus there exists a neighborhood $U_{0}$ of $x$ in $M$ such that : $\forall y \in$ $U_{0}, \mathcal{R}_{N_{0}}(L, w ; y) \subset U$. Another time, we have two cases :

1. either there exist $x_{1} \in U_{0}$ and $\left(x_{1}, v_{1}\right) \in \mathcal{R}_{N_{0}}\left(L, w ; x_{1}\right)$ such that $\left(x_{1}, v_{1}\right) \notin$ $\mathcal{N}^{T}(L) ; \mathcal{N}^{T}(L)$ being closed, we have the conclusion for the set $U^{\prime}=U \backslash \mathcal{N}^{T}(L)$.

2. or $\bigcup_{y \in U_{0}} \mathcal{R}_{N_{0}}(L, w ; y) \subset \mathcal{N}^{T}(L)$; then by proposition 20 , $\bigcup_{y \in U_{0}} \mathcal{R}_{N_{0}}(L, w ; y)$ is a union of periodic orbits with period $N_{0}$ contained in $\mathcal{A}^{T}(L)$. These orbits are graphs above a part of the zero section, and have no conjugate point.

\section{Green bundles, conjugate points and proofs of of theorem 2 and corollary 3}

All the results contained in this section except the last proposition are not new.

Let us recall some definitions :

Definition. Let $L$ be a Tonelli Lagrangian function defined on $T M$ and $(x, v) \in T M$ :

- the "vertical" at $(x, v) \in T M$ is the linear subspace $V(x, v)=\operatorname{ker} D \pi(x, v)$ of $T_{(x, v)}(T M)$; the vertical at $(x, p) \in T^{*} M$ is the (Lagrangian) linear subspace $V(x, p)=\operatorname{ker} D \pi^{*}(x, p)=D \mathcal{L}_{L}(V(x, v))$ of $T_{(x, p)}\left(T^{*} M\right) ;$

- the orbit of $(x, v)$ has a conjugate point if there exists $t \neq t^{\prime}$ such that $D f_{t-t^{\prime}}^{L}\left(V\left(f_{t^{\prime}}^{L}(x, v)\right)\right) \cap$ $V\left(f_{t}^{L}(x, v)\right) \neq\{0\}$; then we say that $t$ and $t^{\prime}$ are conjugate (along the orbit); the definition is the same for $(x, p)$.

We recall some results of [7] :

Proposition 23 Let $(x, v)=\left(\gamma\left(t_{0}\right), \dot{\gamma}\left(t_{0}\right)\right)$ be a point of a ray $\gamma: \mathbb{R} \rightarrow M$ for $L$; then its orbit has no conjugate point.

Proposition 24 Let $(x, v)$ be a point of TM which is not a fixed point of the flow $\left(f_{t}^{L}\right)$ and which has no conjugate point; then there exists two $\left(f_{t}^{L}\right)$ invariant $n$-dimensional subbundles of $T(T M), G_{-}$and $G_{+}$, named the Green bundles defined by :

$$
G_{-}(x, v)=\lim _{t \rightarrow+\infty} D f_{-t}^{L}\left(V\left(\phi_{t}(x, v)\right)\right) \quad \text { and } \quad G_{+}(x, v)=\lim _{t \rightarrow+\infty} D f_{t}^{L}\left(V\left(f_{-t}^{L}(x, v)\right)\right) .
$$


Moreover, they are transverse to the vertical and if we define : $\mathbb{L}_{-}(x, v)=D \mathcal{L}(x, v)\left(G_{-}(x, v)\right)$ and $\mathbb{L}_{+}(x, v)=D \mathcal{L}(x, v)\left(G_{+}(x, v)\right)$, then $: \mathbb{L}_{-}(x, v)$ and $\mathbb{L}_{+}(x, v)$ are Lagrangian, their sum is contained in the tangent bundle of the energy level of $\mathcal{L}(x, v)$ and their intersection contains the Hamiltonian vector field.

Proposition 25 Let $(x, v)$ be a T-periodic point with no conjugate point of $\left(f_{t}^{L}\right)$ which is not a fixed point of the flow. Then, if the dimension of $G_{-}(x, v)+G_{+}(x, v)$ is $2 n-$ 1 , this orbit is hyperbolic and for every vector $W \in T_{(x, v)}(T M) \backslash\left(G_{-}(x, v) \cap G_{+}(x, v)\right)$ (where $G_{-}(x, v)$ and $G_{+}(x, v)$ designate the Green bundles) : the family $\left(D f_{t}^{L}(x, v)(W)\right)_{t \in \mathbb{R}}$ is unbounded.

Now we shall give a detailed description of the images of the vertical; to do that we need to introduce some new notions :

Definition. Let $\mathbb{L}_{1}, \mathbb{L}_{2}$ be two Lagrangian subspaces of $T_{(x, p)}\left(T^{*} M\right)$ which are transverse to the vertical. Then the restrictions of $D \pi^{*}(x, p): T_{(x, p)}\left(T^{*} M\right) \rightarrow T_{x} M$ to $\mathbb{L}_{1}$ and $\mathbb{L}_{2}$ are two isomorphisms, named $F_{1}$ and $F_{2}$.

The relative height between $\mathbb{L}_{1}$ and $\mathbb{L}_{2}$ is then the quadratic form $Q\left(\mathbb{L}_{1}, \mathbb{L}_{2}\right)$ defined on $T_{x} M$ by :

$$
\forall \delta x \in T_{x} M, Q\left(\mathbb{L}_{1}, \mathbb{L}_{2}\right)(\delta x)=\omega\left(F_{1}^{-1}(\delta x), F_{2}^{-1}(\delta x)\right) .
$$

We say that $\mathbb{L}_{2}$ is above $\mathbb{L}_{1}$ if $Q\left(\mathbb{L}_{1}, \mathbb{L}_{2}\right)$ is positive (i.e. if its index is 0 ), that $\mathbb{L}_{2}$ is strictly above $\mathbb{L}_{1}$ if $\mathbb{L}_{2}$ is above $\mathbb{L}_{1}$ and the dimension of $\mathbb{L}_{1} \cap \mathbb{L}_{2}$ is 0 i.e. if $Q\left(\mathbb{L}_{1}, \mathbb{L}_{2}\right)$ is positive definite and that $\mathbb{L}_{2}$ is semi-strictly above $\mathbb{L}_{1}$ if $\mathbb{L}_{2}$ is above $\mathbb{L}_{1}$ and the dimension of $\mathbb{L}_{1} \cap \mathbb{L}_{2}$ is 1 i.e. if $Q\left(\mathbb{L}_{1}, \mathbb{L}_{2}\right)$ is positive with nullity 1 .

REMARK. The definition of the height (slightly different because given in a chart) was given in [2].

Let us recall some results of [7] and [2] :

Proposition 26 We define : $V_{t}(x, p)=D \Phi_{t}^{H}\left(V\left(\Phi_{-t}^{H}(x, p)\right)\right.$. Then :

- Let $\mathbb{L}$ be a Lagrangian subspace of $T_{(x, p)}\left(T^{*} M\right)$ which is transverse to $V(x, p)$; for $t>0$ small enough : $V_{t}(x, p)$ is strictly above $\mathbb{L}$ which is strictly above $V_{-t}(x, p)$ ("small enough" is locally uniform in $(x, p)$ );

- if $0<t_{1}<t_{2}$ and the orbit has no conjugate point between 0 and $t_{2}: V_{t_{1}}(x, p)$ is strictly above $V_{t_{2}}(x, p)$;

- if $0<t_{1}<t_{2}$ and the orbit has no conjugate point between $-t_{2}$ and $0: V_{-t_{2}}(x, p)$ is strictly above $V_{-t_{1}}(x, p)$;

- if $t, t^{\prime}$ are strictly positive and the orbit has no conjugate point between $-t$ and $t^{\prime}$, then $V_{t^{\prime}}(x, p)$ is strictly above $V_{-t}(x, p)$. 
A first consequence is the well-known :

Corollary 27 Let $(x, p)$ be a point having no conjugate point for $H$; then $\mathbb{L}_{+}(x, p)$ is above $\mathbb{L}_{-}(x, p)$.

Another consequence is :

Corollary 28 Let $H: T^{*} M \rightarrow \mathbb{R}$ be a Tonelli Hamiltonian function. Then the subset $\mathcal{U}$ of $C^{\infty}(M) \times T^{*} M$ defined by $: \mathcal{U}=\left\{(\psi,(x, p)) \in C^{\infty}(M) \times T^{*} M\right.$; there are two conjugate points for $H-\psi$ along the orbit of $(x, p)\}$ is open.

Proof of COROLlary 28: Let us assume that there exist some conjugate points along the orbit of $(x, p)$ for $H$ : there exist two real numbers $t_{1}<t_{2}$ such that $D \Phi_{t_{2}-t_{1}}^{H}\left(V\left(\phi_{t_{1}}^{H}(x, p)\right)\right) \cap V\left(\Phi_{t_{2}}^{H}(x, p)\right)$ contains at least one non zero vector, named $Y$. To simplify the notations, we may assume that $t_{1}=0$ and $t_{2}=T>0$. We may assume too that $T$ is the smallest $t>0$ such that 0 and $t$ are conjugate along the orbit of $(x, p)$. We have :

- if $X$ is a non zero vector belonging to $V(x, p) \cap V_{-T}(x, p)$ and if $Y=D \Phi_{T}^{H}(x, p) X$, for $u>0, Z=D \Phi_{-u-T}^{H}(Y)=D \Phi_{-u}^{H}(X) \in V_{-T-u}\left(\Phi_{-u}^{H}(x, p)\right) \cap V_{-u}\left(\Phi_{-u}^{H}(x, p)\right)$.

- for $u>0$ small enough : $Z \in V_{-u}\left(\Phi_{-u}^{H}(x, p)\right)$, the Lagrangian subspace $V_{-2 u}\left(\Phi_{-u}^{H}(x, p)\right)$ is strictly above $V_{-u}\left(\Phi_{-u}^{H}(x, p)\right.$ ) (it is the third point of proposition 26); we choose then $u$ such that $u<T$; then we have : $V_{-T-u}\left(\Phi_{-u}^{H}(x, p)\right)$ is not above $V_{-2 u}\left(\Phi_{-u}^{H}(x, p)\right)$ : indeed, $Z$ belongs to $V_{-T-u}\left(\Phi_{-u}^{H}(x, p)\right)$ and to a Lagrangian subspace, $V_{-u}\left(\Phi_{-u}^{H}(x, p)\right)$, which is "strictly under" $V_{-2 u}\left(\Phi_{-u}^{H}(x, p)\right)$.

Finally, we have found $\left(x_{1}, p_{1}\right)$ on the orbit of $(x, p)$ and $0<t_{1}<t_{2}$ such that $V_{-t_{2}}\left(x_{1}, p_{1}\right)$ is not above $V_{-t_{1}}\left(x_{1}, p_{1}\right)$; this condition is clearly open and implies the existence of conjugate points (see proposition 26).

Let us now prove proposition 5 :

Proposition 5 For the Tonelli Lagrangian functions, the following property is generic: "if $P$ is a periodic orbit which is a graph above a part of the zero section, which has no conjugate point and whose period is an integer $N \geq 1$, then:

- either $P$ is hyperbolic and isolated among the $N$-periodic orbits;

- or in every neighborhood of $P$ there exists an open subset of points whose orbit has conjugate points".

In the proof of proposition 5, we will prove the following result, which is the main ingredient of the proof : 
Proposition 29 Let $L: T M \rightarrow \mathbb{R}$ be a Tonelli Lagrangian functions and $P$ be a non hyperbolic periodic orbit of $\left(f_{t}^{L}\right)$ which is a graph above a part of the zero section, which has no conjugate point and whose period is an integer $N \geq 1$, then :

in every neighbourhood of 0 in $C^{\infty}(M, \mathbb{R})$, there exists a function $\psi$ such that $P$ is a periodic orbit for $\left(f_{t}^{L+\psi}\right)$ with conjugate points.

Proof of Proposition 5: Let $H: T^{*} M \rightarrow \mathbb{R}$ be a Tonelli Hamiltonian function. Let $\left(U_{n}\right)$ be a countable basis of open and relatively compact subsets of $T^{*} M$. The subset $\mathcal{U}_{n}$ of $C^{\infty}(M)$ is the set of $\psi$ such that $U_{n}$ contains a point whose orbit under $\left(\Phi_{t}^{H-\psi}\right)$ has a conjugate point. We deduce from corollary 28 that $\mathcal{U}_{n}$ is open. Therefore $\mathcal{V}_{n}=\mathcal{U}_{n} \cup\left(C^{\infty}(M) \backslash \overline{\mathcal{U}}_{n}\right)$ is an open and dense subset of the Baire space $C^{\infty}(M)$ and $\mathcal{G}=\bigcap_{n \in \mathbb{N}} \mathcal{V}_{n}$ is a dense $G_{\delta}$ subset of $C^{\infty}(M)$.

Let us consider $\psi \in \mathcal{G}$ and let $(x, p)$ be a $N$-periodic point for $\left(\Phi_{t}^{H-\psi}\right)$ whose orbit is a graph above a part of the zero section, which has no conjugate point. Let us assume that there exists a neighborhood $U_{n}$ of $(x, p)$ which contains no point whose orbit under $\left(\Phi_{t}^{H-\psi}\right)$ has a conjugate point. As $\psi \in \mathcal{G}$ and $\psi \notin \mathcal{U}_{n}$, we have : $\psi \in C^{\infty}(M) \backslash \overline{\mathcal{U}}_{n}$.

Let us now consider the orbit of $(x, p)$ : as it has no conjugate points, we can define the Green bundles $\mathbb{L}_{-}$and $\mathbb{L}_{+}$along this orbit. There are two cases :

1) If these Green bundles are transverse in the energy level, we use proposition 25 : $\mathbb{L}_{-}(x, p) \cap \mathbb{L}_{+}(x, p)=\mathbb{R} X_{H}(x, p)$ where $X_{H}$ is the Hamiltonian vector field, the orbit is hyperbolic and the eigenvectors of $D \Phi_{N}^{H}(x, p)$ associated to the eigenvalue 1 are the vectors of $\mathbb{R} X_{H}(x, p)$ (because the orbits of the other vectors are unbounded); it implies that this orbit is isolated among the $N$ periodic orbits.

2) If the Green bundles are not transverse in the energy level, we shall show that we may add to $H-\psi=\tilde{H}$ a small function $\psi_{1} \in C^{\infty}(M)$ to create conjugate points along the orbit of $(x, p)$; it will imply that $\psi \in \overline{\mathcal{U}}_{n}$, it is a contradiction with $\psi \in C^{\infty}(M) \backslash \overline{\mathcal{U}}_{n}$.

Let us now build such a $\psi_{1}$. We assume that $(x, p)$ is not a fixed point of the flow (this case is simpler that the case which we treat); then there exists $t_{0} \geq 0$ such that, if we define : $\gamma(t)=\pi^{*} \circ \Phi_{t}^{\tilde{H}}(x, p)$, then $\dot{\gamma}\left(t_{0}\right) \neq 0$; we define : $x_{0}=\gamma\left(t_{0}\right)$. We choose $C^{\infty}$-coordinates $\left(x^{1}, \ldots, x^{n}\right)$ in a neighborhood $U \subset M$ of $x_{0}$ such that if $U \cap \gamma=\{\gamma(t) ; t \in] t_{0}-\varepsilon, t_{0}+\varepsilon[\}$, then: $\left.\forall t \in\right] t_{0}-\varepsilon, t_{0}+\varepsilon\left[,\left(x^{1}, \ldots, x^{n}\right)(\gamma(t))=(t, 0 \ldots, 0)\right.$. We work then in the dual (symplectic) coordinates $\left(x^{1}, \ldots, x^{n}, p^{1}, \ldots, p^{n}\right)$ on $T^{*} U$ : it means that the point with coordinates $\left(x^{1}, \ldots, x^{n}, p^{1}, \ldots p^{n}\right)$ is $\sum_{k=1}^{n} p^{k} d x^{k}$. We define a function $\psi_{1}: M \rightarrow \mathbb{R}$ which is:

- zero on $M \backslash U$;

- defined in the chart $U$ by: $\psi_{1}(x)=\eta\left(\sum_{i=1}^{n}\left(x^{i}\right)^{2}\right) \sum_{j=2}^{n}\left(x^{j}\right)^{2}$ where $\eta: \mathbb{R} \rightarrow[0,1]$ 
is a $C^{\infty}$ function which is zero outside $]-\left(\frac{\varepsilon}{2}\right)^{2},\left(\frac{\varepsilon}{2}\right)^{2}[$ and strictly positive in ]$-\left(\frac{\varepsilon}{2}\right)^{2},\left(\frac{\varepsilon}{2}\right)^{2}[$.

Then $(x, p)$ has the same (periodic) orbit $\Gamma$ for $\left(\Phi_{t}^{\tilde{H}}\right)$ as for $\left(\Phi_{t}^{\tilde{H}+\psi_{1}}\right)$.

Let us now assume that the orbit of $(x, p)$ has no conjugate point for $\left(\Phi_{t}^{\tilde{H}+\psi_{1}}\right)$ (we shall show a contradiction). Then we may define along this orbit the Green bundles $\mathbb{L}_{-}^{1}$ and $\mathbb{L}_{+}^{1}$ (for $\left.\tilde{H}+\psi_{1}\right)$. We shall use :

Lemma 30 We consider $(x, p) \in \Gamma, \tau>0$ and $\mathbb{L}$ a Lagrangian subspaces of $T_{(x, p)}\left(T^{*} M\right)$ transverse to $V(x, p)$ such that:

a) for every $t \in[0, \tau], D \phi_{t}^{\tilde{H}}(\mathbb{L})$ is transverse to $V\left(\phi^{\tilde{H}}(x, p)\right)$;

b) for every $t \in[0, \tau], D \phi_{t}^{\tilde{H}+\psi_{1}}(\mathbb{L})$ is transverse to $V\left(\phi^{\tilde{H}}(x, p)\right)$.

Then for every $t \in[0, \tau], D \phi_{t}^{\tilde{H}}(\mathbb{L})$ is above (semi-strictly above if $\left.t \geq N\right) D \phi_{t}^{\tilde{H}+\psi_{1}}(\mathbb{L}$ ).

Proof of LEMma 30: We begin by proving a version of this lemma for small $t$.

We say that $(\delta x, \delta p): \mathbb{R} \rightarrow T\left(T^{*} M\right)$ is an infinitesimal solution along the orbit of $(x, p)$ for $\left(\phi_{t}\right)$ if $(\delta x(t), \delta p(t)) \in T_{\phi_{t}(x, p)}\left(T^{*} M\right)$ and $(\delta x(t), \delta p(t))=D \phi_{t}(\delta x(0), \delta p(0))$. Let $\left(\delta x_{1}, \delta p_{1}\right)$ (resp. $\left.\left(\delta x_{0}, \delta p_{0}\right)\right)$ be an infinitesimal solution for $\tilde{H}+\psi_{1}$ (resp. $\left.\tilde{H}\right)$ along $\Gamma$. They satisfy the so-called linearized Hamilton equations (given in coordinates):

$$
\begin{gathered}
\delta \dot{x}_{1}=\frac{\partial^{2} \tilde{H}}{\partial x \partial p} \delta x_{1}+\frac{\partial^{2} \tilde{H}}{\partial p^{2}} \delta p_{1} ; \quad \delta \dot{p}_{1}=-\frac{\partial^{2} \tilde{H}}{\partial x^{2}} \delta x_{1}-\frac{\partial^{2} \tilde{H}}{\partial p \partial x} \delta p_{1}-\frac{\partial^{2} \psi_{1}}{\partial x^{2}}(x) \delta x_{1} ; \\
\delta \dot{x}_{0}=\frac{\partial^{2} \tilde{H}}{\partial x \partial p} \delta x_{0}+\frac{\partial^{2} \tilde{H}}{\partial p^{2}} \delta p_{0} ; \quad \delta \dot{p}_{0}=-\frac{\partial^{2} \tilde{H}}{\partial x^{2}} \delta x_{0}-\frac{\partial^{2} \tilde{H}}{\partial p \partial x} \delta p_{0} .
\end{gathered}
$$

We are interested in some infinitesimal solutions having the same initial values: $\left(\delta x_{0}(0), \delta p_{0}(0)\right)=$ $\left(\delta x_{1}(0), \delta p_{1}(0)\right)$. We deduce from the linearized Hamilton equations that, uniformly for $(x, p) \in \Gamma$ close to $\left(x_{0}, p_{0}\right)$, if the two infinitesimal solutions have the same initial values, for $t$ close to 0 :

$$
(*) \quad \delta x_{1}(t)=\delta x_{0}(t)+O\left(t^{2}\right) ; \quad \delta p_{1}(t)=\delta p_{0}(t)-t \frac{\partial^{2} \psi_{1}}{\partial x^{2}}(x) \delta x_{1}(t)+O\left(t^{2}\right) .
$$

Let us assume that we work in a dual chart and that $\mathbb{L}$ is a Lagrangian subspace of $T_{(x, p)}\left(T^{*} M\right)$ transverse to $V(x, p)$ : then $\mathbb{L}$ is the graph of a symmetric matrix $S$ : $\delta p=S \delta x$. In this chart, the coordinates of $\Phi_{t}^{\tilde{H}}(x, p)$ are $(x(t), p(t))$. For $t$ small enough $D \Phi_{t}^{\tilde{H}} \mathbb{L}=\mathbb{L}_{t}$ and $D \Phi_{t}^{\tilde{H}+\psi_{1}} \mathbb{L}=\mathbb{L}_{t}^{1}$ are Lagrangian subspace of $T_{\Phi_{t}^{\tilde{H}}(x, p)}\left(T^{*} M\right)$ which are transverse to the vertical : they are graphs of $S_{t}, S_{t}^{1}$. We distinguish two cases (even if they are not exhaustive) : 
a) there exists $\alpha>0$ such that the support of $\psi$ doesn't meet $\{x(t) ; t \in] 0 ; \alpha[\}$; in this case, for every $t \in[0, \alpha], D \phi_{t}^{\tilde{H}}(\mathbb{L})=D \phi_{t}^{\tilde{H}+\psi_{1}}(\mathbb{L})$;

b) there exists $\alpha>0$ such that $\{x(t) ; t \in[0 ; \alpha]\}$ is in the interior of the support of $\psi_{1}$; let $\left(\delta x_{0}, \delta p_{0}\right)$ and $\left(\delta x_{1}, \delta p_{1}\right)$ be some infinitesimal solutions as before such that : $\left(\delta x_{0}(0), \delta p_{0}(0)\right)=\left(\delta x_{1}(0), \delta p_{1}(0)\right) \in \mathbb{L}$; we have :

$$
\begin{gathered}
\delta x_{1}(t)=\delta x_{0}(t)+O\left(t^{2}\right) ; \quad \delta p_{0}(t)=S_{t}(x, p) \delta x_{0}(t) ; \quad \delta p_{1}(t)=S_{t}^{1}(x, p) \delta x_{1}(t) \\
\delta p_{1}(t)=\delta p_{0}(t)-t \frac{\partial^{2} \psi_{1}}{\partial x^{2}}(x) \delta x_{1}(t)+O\left(t^{2}\right)=\left(S_{t}(x, p)-t \frac{\partial^{2} \psi_{1}}{\partial x^{2}}(x)\right) \delta x_{1}(t)+O\left(t^{2}\right)
\end{gathered}
$$

We deduce that : $S_{t}^{1}(x, p)=S_{t}(x, p)-t \frac{\partial^{2} \psi_{1}}{\partial x^{2}}(x)+O\left(t^{2}\right)$; therefore : $S_{t}(x, p)-$ $S_{t}^{1}(x, p)$ a symmetric matrix which is positive with nullity 1 for $t>0$ small enough. It is the matrix (in the chart) of the relative eight $Q\left(D \phi_{t}^{\tilde{H}+\psi_{1}}(\mathbb{L}), D \phi_{t}^{\tilde{H}}(\mathbb{L})\right)$; thus, $D \phi_{t}^{\tilde{H}}(\mathbb{L})$ is semi-strictly above $D \phi_{t}^{\tilde{H}+\psi_{1}}(\mathbb{L})$ for $t$ small enough.

Let us notice that using a limit, we deduce from the case b) that if $\{x(t) ; t \in] 0 ; \alpha]\}$ is in the interior of the support of $\psi_{1}$, we have the same conclusion : then we have dealt with all the possible cases for $(x, p)$.

Now, to prove the lemma for large $t$, we notice that any symplectic flow preserves the order between Lagrangian subspaces : if $\mathbb{L}_{1}, \mathbb{L}_{2}$ are such that $\mathbb{L}_{2}$ is above $\mathbb{L}_{1}$ and such that for every $t \in[0, \tau], D \phi_{t}\left(\mathbb{L}_{1}\right)$ and $D \phi_{t}\left(\mathbb{L}_{2}\right)$ are transverse to the vertical, then the relative height $Q\left(D \phi_{t}\left(\mathbb{L}_{1}\right), D \phi_{t}\left(\mathbb{L}_{2}\right)\right)$ has a kernel varying continuously with $t$ and whose dimension is constant (it is $\operatorname{dim}\left(D \phi_{T}\left(\mathbb{L}_{1} \cap \mathbb{L}_{2}\right)\right)=\operatorname{dim}\left(\mathbb{L}_{1} \cap \mathbb{L}_{2}\right)$ ); therefore its index is constant.

Let us now prove the first part of lemma 30. We have proved that there exists $\alpha>0$ such that : for every $t \in[0, \alpha], D \phi_{t}^{\tilde{H}}\left(\mathbb{L}_{1}\right)$ is above $D \phi_{t}^{\tilde{H}+\psi_{1}}\left(\mathbb{L}_{1}\right)$. Let us define : $\tau_{1}=\sup \left\{R \in[0, \tau] ; \forall t \in[0, R], Q\left(D \phi_{t}^{\tilde{H}+\psi_{1}}\left(\mathbb{L}_{1}\right), D \phi_{t}^{\tilde{H}}\left(\mathbb{L}_{1}\right)\right) \geq 0\right\}$. Let us assume that $\tau_{1}<\tau$; at first, we notice that, by continuity, the supremum is indeed a maximum : $Q\left(D \phi_{\tau_{1}}^{\tilde{H}+\psi_{1}}\left(\mathbb{L}_{1}\right), D \phi_{\tau_{1}}^{\tilde{H}}\left(\mathbb{L}_{1}\right)\right) \geq 0$, i.e. $D \phi_{\tau_{1}}^{\tilde{H}}\left(\mathbb{L}_{1}\right)$ is above $D \phi_{\tau_{1}}^{\tilde{H}+\psi_{1}}\left(\mathbb{L}_{1}\right)$; because the flow preserves the order between Lagrangian subspaces, we deduce that for any $u \in$ $\left[0, \tau-\tau_{1}\right], D \phi_{\tau_{1}+u}^{\tilde{H}}\left(\mathbb{L}_{1}\right)$ is above $D \phi_{u}^{\tilde{H}}\left(D \phi_{\tau_{1}}^{\tilde{H}+\psi_{1}}\left(\mathbb{L}_{1}\right)\right)$; but for $u>0$ small enough, we have : $D \phi_{u}^{\tilde{H}}\left(D \phi_{\tau_{1}}^{\tilde{H}+\psi_{1}}\left(\mathbb{L}_{1}\right)\right)$ is above $D \phi_{\tau_{1}+u}^{\tilde{H}+\psi_{1}}\left(\mathbb{L}_{1}\right)$; therefore, for $u>0$ small enough : $\tau_{1}+u$ contradicts the definition of $\tau_{1}$.

To obtain the "semi-strict" of the lemma, we notice that along a subarc of the orbit of $(x, p)$ (in the support of $\psi_{1}$ ), we find locally a strict inequality between the Lagrangian subspaces (it is the case b) before).

Using this lemma (the vertical is not transverse to itself, but we may use an image of this vertical), we obtain : 
for every $t>N, D \Phi_{t}^{\tilde{H}}\left(V\left(\Phi_{-t}^{\tilde{H}}(x, p)\right)\right)$ is semi-strictly above $D \Phi_{t}^{\tilde{H}+\psi_{1}}\left(V\left(\Phi_{-t}^{\tilde{H}+\psi_{1}}(x, p)\right)\right)$; when $t$ tends to $+\infty$, we obtain : $\mathbb{L}_{+}(x, p)$ is above $\mathbb{L}_{+}^{1}(x, p)$. As $\mathbb{L}_{+}^{1}(x, p)$ is invariant under $\left(D \Phi_{t}^{\tilde{H}+\psi_{1}}\right)$, we deduce that for every $t>0: D \Phi_{t}^{\tilde{H}+\psi_{1}}\left(\mathbb{L}_{+}\left(\Phi_{-t}^{\tilde{H}+\psi_{1}}(x, p)\right)\right)$ is semi-strictly above $\mathbb{L}_{+}^{1}(x, p)$. But by lemma 30 and the fact that $\mathbb{L}_{+}(x, p)$ is invariant under $\left(D \Phi_{t}^{\tilde{H}}\right)$, we know that, for $t>N, \mathbb{L}_{+}(x, p)$ is semi-strictly above $D \Phi_{t}^{\tilde{H}+\psi_{1}}\left(\mathbb{L}_{+}\left(\Phi_{-t}^{\tilde{H}+\psi_{1}}(x, p)\right)\right)$. Finally, $\mathbb{L}_{+}(x, p)$ is semi-strictly above $\mathbb{L}_{+}^{1}(x, p)$.

In a similar way, we obtain that $\mathbb{L}_{-}^{1}(x, p)$ is semi-strictly above $\mathbb{L}_{-}(x, p)$. Finally, we have :

- $\mathbb{L}_{+}(x, p)$ is semi-strictly above $\mathbb{L}_{+}^{1}(x, p)$, i.e $Q\left(\mathbb{L}_{+}^{1}(x, p), \mathbb{L}_{+}(x, p)\right)$ has a 1-dimension kernel and is positive ;

- $\mathbb{L}_{-}^{1}(x, p)$ is semi-strictly above $\mathbb{L}_{-}(x, p)$ i.e. $Q\left(\mathbb{L}_{-}(x, p), \mathbb{L}_{-}^{1}(x, p)\right)$ has a 1 -dimension kernel and is positive ;

- $\operatorname{dim}\left(\mathbb{L}_{-}(x, p) \cap \mathbb{L}_{+}(x, p) \geq 2\right)$ i.e. $Q\left(\mathbb{L}_{-}(x, p), \mathbb{L}_{+}(x, p)\right.$ is positive and the dimension of its kernel is at least 2 .

Therefore :

$Q\left(\mathbb{L}_{-}^{1}(x, p), \mathbb{L}_{+}^{1}(x, p)\right)=-Q\left(\mathbb{L}_{-}(x, p), \mathbb{L}_{-}^{1}(x, p)\right)+Q\left(\mathbb{L}_{-}(x, p), \mathbb{L}_{+}(x, p)\right)-Q\left(\mathbb{L}_{+}^{1}(x, p), \mathbb{L}_{+}(x, p)\right)$

is strictly negative at any vector of $\operatorname{ker} Q\left(\mathbb{L}_{-}(x, p), \mathbb{L}_{+}(x, p)\right) \backslash \operatorname{ker} Q\left(\mathbb{L}_{+}^{1}(x, p), \mathbb{L}_{+}(x, p)\right)$ and we obtain a contradiction with corollary 27.

Proof of THEOREM 2 :

We explained in the introduction how we deduce the last assertion of theorem 2 fom propositions propositions 4 and 5 .

The first part of the theorem is a consequence of the end of the theorem and a result of Baire's theory :

we consider a generic Tonelli Lagrangian function. The union $\mathcal{R}^{*}$ of the regular level of $H$ is a dense open subset of $\mathbb{R}$. We denote the set of the regular values of $H$ by $V$. Let us consider $h_{0} \in V$; then there exists a diffeomorphism, $\left.\Phi: H^{-1}\left(h_{0}\right) \times\right]-\varepsilon, \varepsilon[\rightarrow$ $U \subset T^{*} M$ such that : $\left.\forall \eta \in\right]-\varepsilon, \varepsilon\left[, \Phi\left(H^{-1}\left(h_{0}\right) \times\{\eta\}\right)=H^{-1}\left(h_{0}+\eta\right)\right.$. Then $A=$ $\Phi^{-1}\left(\overline{\mathcal{A}_{*}^{T}(H)} \cap U\right)$ is a closed subset of $\left.H^{-1}\left(h_{0}\right) \times\right]-\varepsilon, \varepsilon\left[\right.$ which has no interior. Let $\left(U_{n}\right)$ be a basis of non empty subsets of $H^{-1}\left(h_{0}\right)$. We define : $\mathcal{F}_{n}=\{\eta \in]-\varepsilon, \varepsilon\left[; U_{n} \times\{\eta\} \subset\right.$ $\left.\left(H^{-1}\left(h_{0}\right) \times\{\eta\}\right) \cap A\right\}$. As $A$ is closed, $\mathcal{F}_{n}$ is a closed subset of $]-\varepsilon, \varepsilon$. Moreover, as $A$ has no interior, $\mathcal{F}_{n}$ has no interior; therefore $F=\bigcup_{n \in \mathbb{N}} \mathcal{F}_{n}$ has no interior (Baire's theorem) and $G=h_{0}+(]-\varepsilon, \varepsilon[\backslash F)$ is a dense $G_{\delta}$ subset of $] h_{0}-\varepsilon, h_{0}+\varepsilon[$ such that : for every $h \in G, H^{-1}(h) \cap \mathcal{A}_{*}^{T}(H)$ has no interior in $H^{-1}(h)$. 
Proof of COROLlary 3]: We want to prove that the set

$$
W^{s}\left(\overline{\mathcal{A}_{*}^{T}(H)} ;\left(\Phi_{t}^{H}\right)\right) \cup W^{u}\left(\overline{\mathcal{A}_{*}^{T}(H)} ;\left(\Phi_{t}^{H}\right)\right)
$$

has no interior. Let us assume that it is not true and let $U \subset W^{s}\left(\overline{\mathcal{A}_{*}^{T}(H)} ;\left(\Phi_{t}^{H}\right)\right) \cup$ $W^{u}\left(\overline{\mathcal{A}_{*}^{T}(H)} ;\left(\Phi_{t}^{H}\right)\right)$ be an open and non empty subset. Using theorem 2 , we know that the open set $U^{\prime}=U \backslash \overline{\mathcal{A}_{*}^{T}(H)}$ is non empty. By Poincaré recurrence theorem, almost every point in $U^{\prime}$ (for the volume form associated to the symplectic form) is positively and negatively recurrent. But a point of $U^{\prime}$ is in

$$
\left.\left(W^{s}\left(\overline{\mathcal{A}_{*}^{T}(H)} ;\left(\Phi_{t}^{H}\right)\right) \cup W^{u}\left(\overline{\mathcal{A}_{*}^{T}(H)} ;\left(\Phi_{t}^{H}\right)\right)\right) \backslash \overline{\mathcal{A}_{*}^{T}(H)} ;\left(\Phi_{t}^{H}\right)\right) ;
$$

therefore, either it is not negatively recurrent or it is not positively recurrent, which contradicts the fact that $U^{\prime}$ is non empty.

\section{Proof of proposition 6}

We begin by defining a completely integrable Tonelli Hamiltonian function of $T^{*} \mathbb{T}^{2}$, whose flow is the "product" of the flow of a pendulum and the geodesic flow of the circle : if we identify $T^{*} \mathbb{T}^{2}$ with the set $\mathbb{T} \times \mathbb{R} \times \mathbb{T} \times \mathbb{R}$, if the (global) coordinates are $\left(\theta_{1}, p_{1}, \theta_{2}, p_{2}\right) \in \mathbb{T} \times \mathbb{R} \times \mathbb{T} \times \mathbb{R}$, the Hamiltonian function $H_{0}$ is defined by :

$$
H_{0}\left(\theta_{1}, p_{1}, \theta_{2}, p_{2}\right)=\frac{1}{2}\left(p_{1}^{2}+p_{2}^{2}\right)+\cos \left(2 \pi \theta_{1}\right)-\frac{3}{2} ;
$$

then the Hamiltonian flow of $H_{0}$ is defined by : $\Phi_{t}^{H_{0}}\left(\left(\theta_{1}, p_{1}, \theta_{2}, p_{2}\right)=\left(\varphi_{t}\left(\theta_{1}, p_{1}\right), \psi_{t}\left(\theta_{2}, p_{2}\right)\right)\right.$ where $\left(\varphi_{t}\right)$ is the flow of the pendulum and $\left(\psi_{t}\right)$ the geodesic flow of $\mathbb{T}$.

Let $w$ be the cohomological class of the 1 -form $d \theta_{2}$. Then :

$$
\mathcal{M}_{w}^{*}\left(H_{0}\right)=\mathcal{A}_{w}^{*}\left(H_{0}\right)=\mathcal{N}_{w}^{*}\left(H_{0}\right)=\{(0,0, t, 1) ; t \in \mathbb{T}\} .
$$

If we perturb slightly $H_{0}$, we may obtain a Hamiltonian function $H_{1}$ such that :

1. $\mathcal{N}_{w}\left(H_{1}\right)=\mathcal{N}_{w}\left(H_{0}\right)$ is a periodic hyperbolic orbit $P$ (in fact, the Mañé set $\mathcal{N}_{w}(H)$ depends continuously on $H$ );

2. the intersections between the stable manifold $W^{s}\left(P,\left(\Phi_{t}^{H_{1}}\right)\right)$ and the unstable manifold $W^{u}\left(P,\left(\Phi_{t}^{H_{1}}\right)\right)$ are transverse in the energy level $\Sigma=H_{1}^{-1}(0)$ of $P$;

3. the surface $S=\left\{\left(\theta_{1}, p_{1}, 0, p_{2}\right) ; H_{1}\left(\theta_{1}, p_{1}, 0, p_{2}\right)=0\right\}$ is transverse to the flow in the 0 energy level and near the point $(0,0,0,1)$; 
4. in any neighborhood $V$ of $(0,0,0,1)$ in $S$, there exists another neighborhood $U$ of $(0,0,0,1)$ in $S$ such that $U \subset V$ and such that $\delta U=\gamma_{1} \cup \gamma_{2} \cup \gamma_{3} \cup \gamma_{4}$ where the $\gamma_{i}$ are some arcs such that : $\gamma_{1} \cup \gamma_{3} \subset W^{s}\left(P,\left(\Phi_{t}^{H_{1}}\right)\right)$ and $\gamma_{2} \cup \gamma_{4} \subset W^{u}\left(P,\left(\Phi_{t}^{H_{1}}\right)\right)$; to obtain such a result, we only have to ask that there is a transverse homoclinic intersection on any local branch of $W^{s}\left(P,\left(\Phi_{t}^{H_{1}}\right)\right) \cap S$ and $W^{u}\left(P,\left(\Phi_{t}^{H_{1}}\right)\right) \cap S$ : then we obtain a kind of canvas by $\operatorname{arcs}$ of $W^{s}\left(P,\left(\Phi_{t}^{H_{1}}\right)\right) \cap S$ and $W^{u}\left(P,\left(\Phi_{t}^{H_{1}}\right)\right) \cap S$ around $(0,0,0,1)$ in $S$.

The situation which we just described is in fact open in the following sense :

there exist $\varepsilon>0$ and an open subset $\mathcal{U}$ of $C^{\infty}(M)$ containing 0 such that : for every $\psi \in \mathcal{U}$, for every $h \in[-\varepsilon, \varepsilon],\left(H_{1}+\psi\right)^{-1}(h)$ contains one periodic orbit $P^{\prime}=$ $P(\psi, h)$, the orbit of $\xi$, close to $P$ and in any neighborhood $V$ of $\xi$ in $S^{\prime}=S(\psi, h)=$ $\left\{\left(\theta_{1}, p_{1}, 0, p_{2}\right) ;\left(H_{1}+\psi\right)\left(\theta_{1}, p_{1}, 0, p_{2}\right)=h\right\}$, there exists another neighborhood $U$ of $\xi$ in $S^{\prime}$ such that $U \subset V$ and such that $\delta U=\gamma_{1} \cup \gamma_{2} \cup \gamma_{3} \cup \gamma_{4}$ where the $\gamma_{i}$ are some arcs such that : $\gamma_{1} \cup \gamma_{3} \subset W^{s}\left(P^{\prime},\left(\Phi_{t}^{H_{1}+\psi}\right)\right)$ and $\gamma_{2} \cup \gamma_{4} \subset W^{u}\left(P^{\prime},\left(\Phi_{t}^{H_{1}+\psi}\right)\right)$.

Moreover, there exists a neighborhood $\mathcal{V} \subset \mathcal{U}$ of 0 in $C^{\infty}(M)$ such that, for every $\psi \in \mathcal{V}$, there exists $h=h(\psi) \in]-\varepsilon, \varepsilon\left[\right.$ such that $\mathcal{N}_{w}\left(H_{1}+\psi\right)=P(\psi, h)$ (we have seen that the Mañé set depends continuously on $\psi$, and an invariant set contained in a neighborhood of a hyperbolic orbit and in an energy level is necessarily a periodic orbit). Let us prove that for $\psi \in \mathcal{V}, \mathcal{N}_{w}\left(H_{1}+\psi\right)$ is not a subset of $\mathcal{I}\left(H_{1}+\psi\right)$.

Let us consider $\xi \in P(\psi, h) \cap S(\psi, h)$ and let us assume that there exists a sequence of K.A.M. tori $\left(T_{i}\right)_{i \in \mathbb{N}}$ such that $\lim _{i \rightarrow \infty} d\left(\xi, T_{i}\right)=0$. Being a Lagrangian invariant torus, each $T_{i}$ is in an energy level $\left(H_{1}+\psi\right)^{-1}\left(h_{i}\right)$ with, for $i$ large enough : $\left.h_{i} \in\right]-\varepsilon, \varepsilon\left[\right.$ close to $P\left(\psi, h_{i}\right)$. Moreover, $T_{i}$ is a graph above the zero section. Therefore, $\left\{\left(\theta_{1}, p_{1}, 0, p_{2}\right) ;\left(\theta_{1}, p_{1}, 0, p_{2}\right) \in T_{i}\right\}$ is a curve $\Gamma_{i}$ which is a graph above a circle. Moreover, this curve passes very close to the point $\xi_{i}$ which is the point of the periodic orbit $P\left(\psi, h_{i}\right)$ which belongs to $S\left(\psi, h_{i}\right)$. This curve is then a curve which is traced on $S\left(\psi, h_{i}\right)$, which has points very close to $\xi_{i}$ and other points far from $\xi_{i}$. Therefore it cuts the boundary of any sufficiently small neighborhood of $\xi_{i}$ in $S\left(\psi, h_{i}\right)$, and then contains some points of $W^{s}\left(P\left(\psi, h_{i}\right),\left(\Phi_{t}^{H_{1}+\psi}\right)\right) \cup W^{s}\left(P\left(\psi, h_{i}\right),\left(\Phi_{t}^{H_{1}+\psi}\right)\right)$. This contradicts the fact that the restriction of the flow to any K.A.M. torus is minimal. [ 


\section{References}

[1] M.-C. Arnaud, Création de points périodiques de tous types au voisinage des tores K.A.M, Bull. Soc. math. France 123 (1995), 591-603.

[2] M.-C. Arnaud, Hyperbolic periodic orbits and Mather sets in certain symmetric cases, Ergodic Theory Dynam. Systems 26 (2006), no. 4, 939-959.

[3] M. J. Carneiro, On minimizing measures of the action of autonomous Lagrangians, Nonlinearity 8 (1995), no. 6, 1077-1085.

[4] G. Contreras, Action potential and weak KAM solutions, Calc. Var. Partial Differential Equations 13 (2001), no. 4, 427-458.

[5] G. Contreras, J. Delgado \& R. Iturriaga, Lagrangian flows: the dynamics of globally minimizing orbits. II. Bol. Soc. Brasil. Mat. (N.S.) 28 (1997), no. 2, 155-196.

[6] G. Contreras, R. Iturriaga, Global minimizers of autonomous Lagrangians, preprint (2000), 208 pp (enlarged version of 22nd Brazilian Mathematics Colloquium (IMPA), Rio de Janeiro, 1999. 148 pp) : www.cimat.mx/ gonzalo/

[7] G. Contreras \& R. Iturriaga, Convex Hamiltonians without conjugate points, Ergodic Theory Dynam. Systems 19 (1999), no. 4, 901-952.

[8] G. Contreras, R. Iturriaga, G. P. Paternain \& M. Paternain. Lagrangian graphs, minimizing measures and Mañé critical values, GAFA 8 (1998) 788-809.

[9] G. Contreras \& G. Paternain Connecting orbits between static classes for generic Lagrangian systems. Topology 41 (2002), no. 4, 645-666.

[10] A. Fathi, Weak KAM theorems in Lagrangian dynamics, Cours à l'ENS Lyon (2003).

[11] A. Fathi \& A. Siconolfi, Existence of $C^{1}$ critical subsolutions of the HamiltonJacobi equation, Invent. Math. 155 (2004), no. 2, 363-388.

[12] M. Herman, On the dynamics on Lagrangian tori invariant by symplectic diffeomorphisms, Progress in variational methods in Hamiltonian systems and elliptic equations (L'Aquila, 1990), Pitman Res. Notes Math. Ser., 243, Longman Sci. Tech., Harlow, (1992), 92-112.

[13] M. Herman, Sur les courbes invariantes par les difféomorphismes de l'anneau, Vol. 1, Asterisque 103-104 (1983).

[14] R. Mañé, Generic properties and problems of minimizing measures of Lagrangian systems, Nonlinearity 9 (1996), no. 2, 273-310.

[15] R. Mañé, Lagrangian flows: the dynamics of globally minimizing orbits. International Conference on Dynamical Systems (Montevideo, 1995), 120-131, Pitman Res. Notes Math. Ser., 362, Longman, Harlow, (1996). 
[16] J. Mather, Action minimizing invariant measures for positive definite Lagrangian systems, Math. Z. 207 (1991), no. 2, 169-207

[17] J. Mather, Variational construction of connecting orbits, Ann. Inst. Fourier (Grenoble) 43 (1993), no. 5, 1349-1386.

[18] J. Mather, Examples of Aubry sets. Ergodic Theory Dynam. Systems 24 (2004), no. $5,1667-1723$ 